\title{
VARIATIONAL ANALYSIS OF CONVEXLY GENERATED SPECTRAL MAX FUNCTIONS
}

\author{
JAMES V. BURKE AND JULIA EATON
}

\begin{abstract}
The spectral abscissa is the largest real part of an eigenvalue of a matrix and the spectral radius is the largest modulus. Both are examples of spectral max functions - the maximum of a real-valued function over the spectrum of a matrix. These mappings arise in the control and stabilization of dynamical systems. In 2001, Burke and Overton characterized the regular subdifferential of the spectral abscissa and showed that the spectral abscissa is subdifferentially regular in the sense of Clarke when all active eigenvalues are nonderogatory. In this paper we develop new techniques to obtain these results for the more general class of convexly generated spectral max functions. In particular, we extend the Burke-Overton subdifferential regularity result to this class. These techniques allow us to obtain new variational results for the spectral radius.

Dedicated to R. Tyrrell Rockafellar on the occasion of his 80th birthday. We gratefully acknowledge Terry's leadership, guidance and friendship.
\end{abstract}

Key words. spectral function - spectral max functions - spectral radius spectral abscissa - subdifferential regularity - variational analysis - chain rule

\section{INTRODUCTION}

A spectral function maps the space of complex $n$-by- $n$ matrices, $\mathbb{C}^{n \times n}$, to the extended reals $\overline{\mathbb{R}}:=\mathbb{R} \cup\{+\infty\}$ and depends only on the eigenvalues of its argument independent of permutations [11, 16]. Given $f: \mathbb{C} \rightarrow \overline{\mathbb{R}}$, the spectral max function $\mathfrak{f}: \mathbb{C}^{n \times n} \rightarrow \overline{\mathbb{R}}$ generated by $f$ is

$$
\mathfrak{f}(X):=\max \{f(\lambda) \mid \lambda \in \mathbb{C} \text { and } \operatorname{det}(\lambda I-X)=0\} .
$$

Spectral max functions are continuous if the underlying function $f$ is continuous; however, they are generally not Lipschitz continuous. Two important spectral max functions are the spectral abscissa and spectral radius, obtained by setting $f(\cdot)=\operatorname{Re}(\cdot)$ and $f(\cdot)=|\cdot|$, respectively. These functions are connected to classical notions of stability for continuous and discrete dynamical systems. In recent years, much research has been dedicated to understanding the variational properties of the spectral abscissa [3, 4, 5, 6, 7, 10, 11, 16, 17. Burke and Overton [11] develop a formula for the regular subdifferential of the spectral abscissa and establish its subdifferential regularity (see $\$ 2.3$ ) on the set of nonderogatory matrices. Subdifferential regularity allows one to exploit a powerful subdifferential calculus for such functions in order to describe their underlying variational properties. A primary goal of this paper is to extend the Burke-Overton subdifferential regularity

Date: August 15, 2015.

The work of James V. Burke is partially supported by NSF grant DMS- 1514459 . 
result to the class of convexly generated spectral max functions defined below (see Theorem 231).

The variational analysis of spectral max functions builds on that for polynomial root max functions developed in [8, 9, 12]. Let $\mathbb{P}^{n}$ denote the set of complex polynomials of degree $n$ or less in a single variable over $\mathbb{C}$, and let $\mathbb{P}^{[n]}$ be those polynomials of degree precisely $n$. The polynomial root max function generated by $f$ is the mapping $\mathbf{f}: \mathbb{P}^{n} \rightarrow \overline{\mathbb{R}}$ defined by

$$
\mathbf{f}(p):=\max \{f(\lambda) \mid \lambda \in \mathbb{C} \text { and } p(\lambda)=0\} .
$$

We say that $\mathbf{f}$ is convexly generated if the generating function $f$ is proper, convex, and lsc, which we assume throughout.

The results in 8, 9, 12] are extended in 2, where, in particular, the subdifferential regularity of convexly generated polynomial root max functions is established. Subdifferential formulas are given when $f$ satisfies one of the following two conditions (introduced in [9]) at all "active roots" (see 93.2 ):

$$
\begin{gathered}
\text { " } f \text { is quadratic, or } f \text { is } \mathcal{C}^{2} \text { and positive definite at } \lambda " \\
\text { "rspan }(\partial f(\lambda))=\mathbb{C} "
\end{gathered}
$$

where $f^{\prime \prime}(\lambda ; \cdot, \cdot)$ is defined in (10), $\partial f(\lambda)$ is the usual subdifferential from convex analysis, and, for $S \subset \mathbb{C}$, $\operatorname{rspan}(S):=\{\tau \zeta \mid \tau \in \mathbb{R}, \zeta \in S\}$ is the real linear span of the set $S$. Observe that condition (4) can only hold when $f$ is not differentiable. Consequently, (33) and (4) are mutually exclusive.

Following [16, the authors of [1] directly derive necessary conditions on the matrix entries of a subgradient for the spectral max function (see Theorems 15] and 18) using the permutation invariance of spectral max functions and the Arnold form (see 4.1). In the case of the spectral abscissa, 11] establishes a formula for the regular subdifferential using a chain rule applied to the representation $\mathfrak{f}=\mathbf{f} \circ \Phi_{n}$, where $f(\cdot)=\operatorname{Re}(\cdot)$ and $\Phi_{n}: \mathbb{C}^{n \times n} \rightarrow \mathbb{P}^{[n]}$ is the characteristic polynomial map

$$
\Phi_{n}(X)(\lambda):=\operatorname{det}(\lambda I-X) .
$$

However, since the mapping $\Phi_{n}$ contains no information on Jordan structure, this approach does not provide a direct path to showing that the spectral abscissa inherits the subdifferential regularity of the polynomial abscissa in the nonderogatory case. Instead, subdifferential regularity is established using a non-constructive argument and specialized matrix techniques. This approach applies to affinely generated spectral max functions, but all efforts to extend them to general convexly generated spectral max functions have been persistently rebuffed. In this paper, we show that an approach based on a more refined use of Arnold form developed in [5, Theorems 2.2, 2.7] can succeed when all active eigenvalues are nonderogatory.

Two approaches for extending the variational theory to convexly generated spectral max functions are presented. The first uses the Arnold form (see Theorem 5) to develop a framework for the application of a standard chain rule [19, Theorem 10.6] to simultaneously establish both a formula for the subdifferential and subdifferential regularity when all active eigenvalues are nonderogatory (see Theorem [12). The second extends the methods in [11] to develop a formula for the set of regular subdifferentials without a nonderogatory assumption (see Theorem[12). However, we again emphasize that the second approach does not provide a path toward establishing subdifferential regularity when $f$ is not affine. 
The method of proof for the first approach builds on tools developed in [5, 11]. For $\tilde{n} \leqslant n$, we exploit the fact that a polynomial $\tilde{p} \in \mathbb{P}^{\tilde{n}}$ has a local factorization in $\mathbb{P}^{\tilde{n}}$ based at its roots (see (17)). This gives rise to what we call the factorization space $\mathbb{S}_{\tilde{p}}$ and an associated diffeomorphism $F_{\tilde{p}}: \mathbb{S}_{\tilde{p}} \rightarrow \mathbb{P}^{\tilde{n}}$ (see 33.1 ). The key new ingredient is our mapping $G: \mathbb{C} \times \mathbb{C}^{n \times n} \rightarrow \mathbb{S}_{\tilde{p}}$ (see (43)), which takes a matrix $\tilde{X} \in \mathbb{C}^{n \times n}$ to the "active factor" (of degree $\tilde{n} \leqslant n$ ) associated with its characteristic polynomial $\Phi_{n}(\tilde{X})$ (see (28) and (47)). The resulting framework is described by the following diagram.

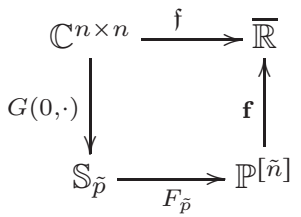

The majority of the paper is devoted to developing the variational properties of the mappings described in this diagram.

Numerous issues need to be addressed in order to place previous work into a comprehensive framework within which modern techniques of nonsmooth analysis apply. The new framework allows us to extend our knowledge of the variational properties of the spectral abscissa to convexly generated spectral max functions. Of the several technical refinements/advances needed for the development of this framework, we describe three of particular significance. First, specialized notions of differentiability are required since the fields on the domain and range of $\mathfrak{f}$ differ (see $\$ 2.2)$. Second, inner products are developed that exploit the local geometry of the spaces $\mathbb{C}^{n \times n}, \mathbb{P}^{\tilde{n}}$, and $\mathbb{S}_{\tilde{p}}$. These inner products give convenient representations for the derivatives of the mappings $G$ and $F_{\tilde{p}}$ and their adjoints (see 2.1 ). Finally, and most importantly, since $\mathfrak{f}$ locally depends only on those eigenvalues that are "active" (see 95 ), the mapping $G$ is introduced to focus the analysis on the eigenspace determined by the active eigenvalues alone, thereby preserving the variational structure required for the application of a standard nonsmooth chain rule.

The paper is organized as follows. In Section 2, we build notation and review the necessary background. In Section 3 we review the polynomial results from $[2$ and 12. In Section 4 we recall the Arnold form and use it to develop a representation for the derivative of the factors of the characteristic polynomial corresponding to nonderogatory eigenvalues. In Section 5 , we derive formulas for the subdifferential and horizon subdifferential of $\mathfrak{f}$ at $\tilde{X}$ when $\tilde{X}$ has nonderogatory active eigenvalues and show the subdifferential regularity of $\mathfrak{f}$ at $\tilde{X}$. In Section 6 , we derive the regular subdifferential of $\mathfrak{f}$ at an arbitrary $\tilde{X} \in \mathbb{C}^{n \times n}$ and show that $\mathfrak{f}$ is subdifferentially regular at $\tilde{X}$ if and only if the active eigenvalues of $\tilde{X}$ are nonderogatory. In Section 7 we apply these results to the spectral radius and illustrate them with two examples. 


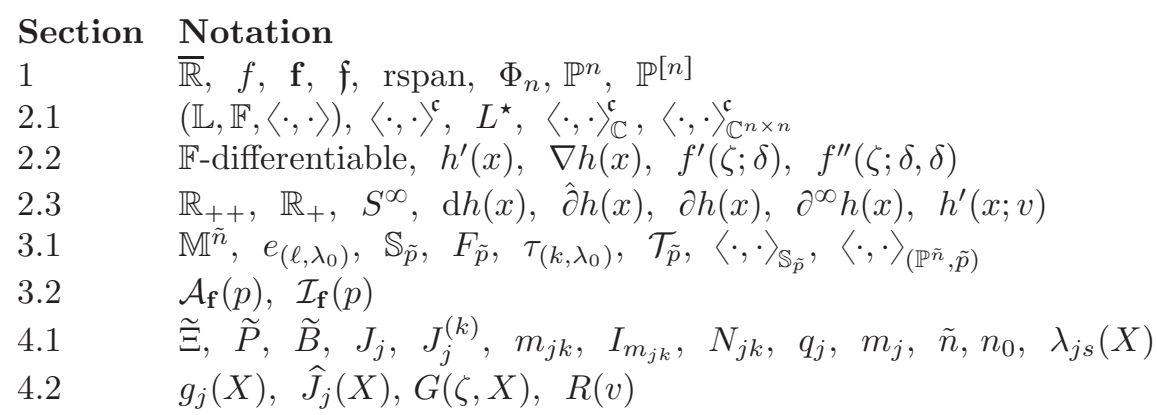

\section{BACKGROUND}

We review inner product spaces, differentiability with respect to a field, and notation and definitions from variational analysis.

2.1. Inner products. A linear space $\mathbb{L}$ over the field $\mathbb{F}$ with real or complex inner product $\langle\cdot, \cdot\rangle$ is called an inner product space, denoted by the triple $(\mathbb{L}, \mathbb{F},\langle\cdot, \cdot\rangle)$. Only the complex $\mathbb{C}$ and real $\mathbb{R}$ fields are considered. When it is important to emphasize that the inner product is complex, we write $\langle\cdot, \cdot\rangle^{\mathfrak{c}}$. A complex inner product space $\left(\mathbb{L}, \mathbb{C},\langle\cdot, \cdot\rangle^{\mathfrak{c}}\right)$ can also be viewed as a real inner product space $(\mathbb{L}, \mathbb{R},\langle\cdot, \cdot\rangle)$ with real inner product $\langle\cdot, \cdot\rangle:=\operatorname{Re}\left(\langle\cdot, \cdot\rangle^{\mathfrak{c}}\right)$. This distinction is important when considering linear transformations and their adjoints, since both depend upon the choice of the underlying field. Recall that if $\operatorname{dim}\left(\mathbb{L}, \mathbb{C},\langle\cdot, \cdot\rangle^{\mathfrak{c}}\right)=n$, then $\operatorname{dim}(\mathbb{L}, \mathbb{R},\langle\cdot, \cdot\rangle)=2 n$.

Let $\mathbb{F}_{0}, \mathbb{F}_{1}, \mathbb{F}_{2}$ be the fields $\mathbb{C}$ or $\mathbb{R}$ with $\mathbb{F}_{0}$ a subfield of both $\mathbb{F}_{1}$ and $\mathbb{F}_{2}$. For $i=1,2$, let $\left(\mathbb{L}_{i}, \mathbb{F}_{i},\langle\cdot, \cdot\rangle_{i}\right)$, be finite-dimensional inner product spaces. Note that both $\mathbb{L}_{1}$ and $\mathbb{L}_{2}$ are inner product spaces over $\mathbb{F}_{0}$ with the appropriate $\mathbb{F}_{0}$ inner product. A transformation $L: \mathbb{L}_{1} \rightarrow \mathbb{L}_{2}$ is said to be $\mathbb{F}_{0}$-linear if for all $\alpha, \beta \in \mathbb{F}_{0}$, $x, y \in \mathbb{L}_{1}$, we have $L(\alpha x+\beta y)=\alpha L(x)+\beta L(y)$. The adjoint mapping $L^{\star}: \mathbb{L}_{2} \rightarrow \mathbb{L}_{1}$ [15] is the unique $\mathbb{F}_{0}$-linear transformation satisfying

$$
\operatorname{Re}\left(\langle x, L y\rangle_{2}\right)=\operatorname{Re}\left(\left\langle L^{\star} x, y\right\rangle_{1}\right) \quad \forall(y, x) \in \mathbb{L}_{1} \times \mathbb{L}_{2} .
$$

As given, this definition appears insufficient since the $\mathbb{F}_{0}$ inner product is not specified in (77); however, this is resolved by noting that the imaginary part of a complex inner product can be obtained from knowledge of only its real part since $\operatorname{Re}\left(\langle\mathrm{i} x, y\rangle^{\mathfrak{c}}\right)=\operatorname{Im}\left(\langle x, y\rangle^{\mathfrak{c}}\right)$, where $\mathrm{i}:=\sqrt{-1}$. That is, the adjoint can be obtained using only the real part of the $\mathbb{F}_{0}$ inner product.

It is essential to be mindful of the distinction between the adjoint of a linear operator and the Hermitian adjoint of a matrix. The adjoint of a linear operator $L$ is denoted by $L^{\star}$, whereas the Hermitian adjoint of a matrix $M \in \mathbb{C}^{n \times m}$ is denoted by $M^{*}$. For example, the following elementary lemma is key.

Lemma 1 (Adjoint of a linear functional). Let $(\mathbb{L}, \mathbb{F},\langle\cdot, \cdot\rangle)$ be a finite dimensional inner product space where $\mathbb{F}$ is either $\mathbb{C}$ or $\mathbb{R}$. Given $y \in \mathbb{L}$, define $L: \mathbb{L} \rightarrow \mathbb{F}$ as the linear functional $L x:=\langle y, x\rangle$ for all $x \in \mathbb{L}$. Then $L^{\star} \zeta=\zeta y$, where the left-hand side shows the action of the adjoint, and the right-hand side is multiplication of $y$ by the scalar $\zeta \in \mathbb{F}$.

The next lemma provides a key tool in our construction of inner products, which in turn impacts the nature of the adjoint operator. 
Lemma 2 (Inner product construction). 2, Lemma 4.1] Let $\mathbb{L}_{1}$ and $\mathbb{L}_{2}$ be finite dimensional vector spaces over $\mathbb{F}=\mathbb{C}$ or $\mathbb{R}$, and let $L: \mathbb{L}_{1} \rightarrow \mathbb{L}_{2}$ be an $\mathbb{F}$-linear isomorphism.

(1) Suppose $\mathbb{L}_{2}$ has inner product $\langle\cdot, \cdot\rangle_{2}$. Then the bilinear form $\mathcal{B}: \mathbb{L}_{1} \times$ $\mathbb{L}_{1} \rightarrow \mathbb{F}$ given by $\mathcal{B}(x, y):=\langle L x, L y\rangle_{2}$ for all $x, y \in \mathbb{L}_{1}$ is an inner product on $\mathbb{L}_{1}$. Denote this inner product by $\langle\cdot, \cdot\rangle_{1}:=\mathcal{B}(\cdot, \cdot)$. Then the adjoint $L^{\star}: \mathbb{L}_{2} \rightarrow \mathbb{L}_{1}$ with respect to the inner products $\langle\cdot, \cdot\rangle_{1}$ and $\langle\cdot, \cdot\rangle_{2}$ equals $L^{-1}$.

(2) Let $\left(\mathbb{L}_{i}, \mathbb{F},\langle\cdot, \cdot\rangle_{i}\right)$ be inner product spaces for $i=1,2$. If $\langle x, y\rangle_{1}=\langle L x, L y\rangle_{2}$ for all $x, y \in \mathbb{L}_{1}$, then $L^{\star}=L^{-1}$ with respect to these inner products.

As a vector space, we endow $\mathbb{C}$ with the complex inner product $\langle\xi, \zeta\rangle_{\mathbb{C}}^{\mathfrak{c}}:=$ $\bar{\xi} \zeta$. This inner product defines the standard complex inner product on $\mathbb{C}^{n+1}$ by $\left\langle\left(a_{0}, a_{1}, \ldots, a_{n}\right),\left(b_{0}, b_{1}, \ldots, b_{n}\right)\right\rangle_{\mathbb{C}^{n+1}}^{\mathfrak{c}}:=\sum_{\ell=0}^{n}\left\langle a_{\ell}, b_{\ell}\right\rangle_{\mathbb{C}}^{\mathfrak{c}}$ for all $a_{\ell}, b_{\ell} \in \mathbb{C}, \ell=$ $0, \ldots, n$. We also work on the space $\mathbb{C}^{n \times n}$ of complex $n \times n$ matrices with complex inner product $\langle X, Y\rangle_{\mathbb{C}^{n \times n}}^{\mathfrak{c}}:=\operatorname{tr}\left(X^{*} Y\right)$. We use Lemma 2 to construct further inner products as needed. In each case, there is a complex version and an associated real version obtained by taking the real part of the complex inner product with $\langle\cdot, \cdot\rangle:=\operatorname{Re}\left(\langle\cdot, \cdot\rangle^{\mathfrak{c}}\right)$, where the bilinearity of the real inner product is considered to be over the scalar field $\mathbb{R}$.

2.2. Derivatives. Again let $\mathbb{F}_{0}, \mathbb{F}_{1}, \mathbb{F}_{2}$ be the fields $\mathbb{R}$ or $\mathbb{C}$ with $\mathbb{F}_{0} \subset \mathbb{F}_{1} \cap \mathbb{F}_{2}$. For $i=1,2$, let $\left(\mathbb{L}_{i}, \mathbb{F}_{i},\langle\cdot, \cdot\rangle_{i}\right)$, be finite-dimensional inner product spaces with associated inner product norms $\|\cdot\|_{i}$. Let $h: \mathbb{L}_{1} \rightarrow \mathbb{L}_{2}$. We say that $h$ is $\mathbb{F}_{0}$ differentiable at $x \in \mathbb{L}_{1}$ if there exists an $\mathbb{F}_{0}$-linear transformation, denoted $h^{\prime}(x)$ : $\mathbb{L}_{1} \rightarrow \mathbb{L}_{2}$, such that

$$
h(y)=h(x)+h^{\prime}(x)(y-x)+o\left(\|y-x\|_{1}\right),
$$

where $\lim _{y \rightarrow x}\left\|o\left(\|y-x\|_{1}\right) /\right\| y-x\left\|_{1}\right\|_{2}=0$. Clearly every $\mathbb{C}$-derivative is an $\mathbb{R}$ derivative, but the converse is false. If $h: \mathbb{L}_{1} \rightarrow \mathbb{F}_{0}, h^{\prime}(x)$ defines an linear functional from $\mathbb{L}_{1}$ into $\mathbb{F}_{0}$ and, since $\mathbb{L}_{1}$ is a vector space over $\mathbb{F}_{0}$, there exists an element of $\mathbb{L}_{1}$, denoted by $\nabla h(x)$, satisfying

$$
h^{\prime}(x) z=\left\{\begin{array}{ll}
\operatorname{Re}\langle\nabla h(x), z\rangle_{1} & \text { if } \mathbb{F}_{0}=\mathbb{R} \\
\langle\nabla h(x), z\rangle_{1} & \text { if } \mathbb{F}_{0}=\mathbb{C}
\end{array} \quad \forall z \in \mathbb{L}_{1} .\right.
$$

We call $\nabla h(x)$ the gradient of $h$ at $x$, and recall from our previous discussion that the vector $\nabla h(x)$ is the same element of $\mathbb{L}_{1}$ regardless of whether $\mathbb{F}_{0}$ equals $\mathbb{C}$ or $\mathbb{R}$. Lemma 1 tells us that

$$
h^{\prime}(x)^{\star} \zeta=\zeta \nabla h(x)
$$

Since we make extensive use of $\mathbb{R}$-differentiability for real-valued functions on $\mathbb{C}$, following [11], we show how to construct the $\mathbb{R}$-derivative in this case. Define the $\mathbb{R}$-linear transformation $\Theta: \mathbb{R}^{2} \rightarrow \mathbb{C}$ by $\Theta\left(x_{1}, x_{2}\right):=x_{1}+\mathrm{i} x_{2}$. The inverse mapping is $\Theta^{-1}(\zeta)=(\operatorname{Re}(\zeta), \operatorname{Im}(\zeta))$. Since $\operatorname{Re}\left(\left\langle\zeta, \Theta\left(x_{1}, x_{2}\right)\right\rangle\right)=\left\langle\Theta^{-1}(\zeta),\left(x_{1}, x_{2}\right)\right\rangle$ for all $\zeta \in \mathbb{C}$ and $\left(x_{1}, x_{2}\right) \in \mathbb{R}^{2}$, we have $\Theta^{\star}=\Theta^{-1}$. Given $f: \mathbb{C} \rightarrow \mathbb{R}$, define $\tilde{f}: \mathbb{R}^{2} \rightarrow \mathbb{R}$ by $\tilde{f}:=f \circ \Theta$. If $\tilde{f}$ is differentiable over $\mathbb{R}$ in the usual sense, then, by the chain rule, $f$ is $\mathbb{R}$-differentiable with gradient $\nabla f(\zeta)=\Theta \nabla \tilde{f}\left(\Theta^{-1} \zeta\right)$, and this is consistent with the notation in (8). In [9] this is called differentiable in the real sense. We say that $f$ is twice $\mathbb{R}$-differentiable if $\tilde{f}$ is twice differentiable over $\mathbb{R}$ in the usual sense. In this case, a further application of the chain rule yields $f(\zeta+\delta)=$ 
$f(\zeta)+\langle\nabla f(\zeta), \delta\rangle+(1 / 2)\left\langle\delta, \nabla^{2} f(\zeta) \delta\right\rangle+o\left(|\delta|^{2}\right)$, where $\nabla^{2} f(\zeta):=\Theta \nabla^{2} \tilde{f}\left(\Theta^{-1} \zeta\right) \Theta^{-1}$. Since we only use $\mathbb{R}$-differentiability of functions $f: \mathbb{C} \rightarrow \mathbb{R}$, we simply say that $f$ is differentiable. We also make use of the following notation:

$$
f^{\prime}(\zeta ; \delta):=\langle\nabla f(\zeta), \delta\rangle \quad \text { and } \quad f^{\prime \prime}(\zeta ; \delta, \delta):=\left\langle\delta, \nabla^{2} f(\zeta) \delta\right\rangle
$$

where it follows that $f^{\prime}(\zeta ; \delta)=\lim _{t \downarrow 0}(f(\zeta+t \delta)-f(\zeta)) / t$. We say that $\nabla^{2} f(\zeta)$ is positive definite if $\left\langle\delta, \nabla^{2} f(\zeta) \delta\right\rangle>0$ for all $\delta \neq 0$. We say $f$ is quadratic if $\nabla^{2} f(\zeta)$ is constant in $\zeta$, and we say that $f$ is $\mathcal{C}^{2}$ at $\tilde{\lambda}$ if the map $\lambda \mapsto \nabla^{2} f$ is continuous at $\tilde{\lambda}$. For example, the function $r_{2}(\zeta):=|\zeta|^{2} / 2$ used in [2, Section 7] and Section 7 below is quadratic with $\nabla r_{2}(\zeta)=\zeta$ and $\nabla^{2} r_{2}(\zeta)$ being the identity map on $\mathbb{C}$. This notation clarifies the key hypotheses (3) and (4).

2.3. Variational analysis review. We use the techniques of variational analysis from [13, 18, 19. Let $\left(\mathbb{L}, \mathbb{C},\langle\cdot, \cdot\rangle^{\mathfrak{c}}\right)$ be a finite-dimensional inner product space with associated real inner product $\langle\cdot, \cdot\rangle:=\operatorname{Re}\langle\cdot, \cdot\rangle^{\mathfrak{c}}$. Let $C$ be a non-empty subset of $\mathbb{L}$. The tangent cone to $C$ at a point $x \in C$ is

$$
T_{C}(x):=\left\{d \mid \exists\left\{x^{\nu}\right\} \subset C,\left\{t_{\nu}\right\} \subset \mathbb{R}_{++} \text {s.t. } x^{\nu} \rightarrow x, t_{\nu} \downarrow 0 \text { and } t_{\nu}^{-1}\left(x^{\nu}-x\right) \rightarrow d\right\},
$$

where $\mathbb{R}_{++}:=(0, \infty)$. The tangent cone is a closed subset of $\mathbb{L}[19$, Proposition 6.2]. The regular normal cone to $C$ at a point $x \in C$ is

$$
\widehat{N}_{C}(x):=\left\{z \mid\langle z, v\rangle \leqslant 0 \text { for all } v \in T_{C}(x)\right\} .
$$

Given $S \subset \mathbb{L}$, the horizon cone of $S$ is

$$
S^{\infty}:=\left\{z \in \mathbb{L} \mid \exists\left\{x^{\nu}\right\} \subset S,\left\{t_{\nu}\right\} \subset \mathbb{R}_{+} \text {s.t. } t_{\nu} \downarrow 0 \text { and } t_{\nu} x^{\nu} \rightarrow z\right\},
$$

where $\mathbb{R}_{+}:=[0, \infty)$. The horizon cone is always a nonempty closed cone. If $S$ is convex, $S^{\infty}$ is the usual recession cone from convex analysis. Let $h: \mathbb{L} \rightarrow \overline{\mathbb{R}}$. The essential domain of $h$ is

$$
\operatorname{dom}(h):=\{x \in \mathbb{L} \mid h(x)<\infty\} .
$$

The function $h$ is said to be proper if $\operatorname{dom}(h) \neq \varnothing$. The epigraph of $h$ is given by

$$
\operatorname{epi}(h):=\{(x, \beta) \in \mathbb{L} \times \mathbb{R} \mid h(x) \leqslant \beta\} .
$$

The subderivative of $h$ is the map $\mathrm{d} h(x): \mathbb{L} \rightarrow \overline{\mathbb{R}} \cup\{-\infty\}$ given by

$$
\mathrm{d} h(x)(\tilde{v}):=\liminf _{t \downarrow 0, v \rightarrow \tilde{v}}(h(x+t v)-h(x)) / t .
$$

By [19, Theorem 8.2], the subderivative and the tangent cone to the epigraph are related by epi $(\mathrm{d} h(x))=T_{\mathrm{epi}(h)}(x, h(x)) \forall x \in \operatorname{dom}(h)$. The regular subdifferential of $h$ at $x \in \operatorname{dom}(h)$ is the set of regular subgradients:

$$
\hat{\partial} h(x):=\{z \mid h(y) \geqslant h(x)+\langle z, y-x\rangle+o(\|y-x\|) \quad \forall y \in \mathbb{L}\} .
$$

The regular subdifferential is a closed, convex subset of $\mathbb{L}$. By [19, Theorem 8.9], the regular subdifferential and the regular normal cone to the epigraph are related by $\hat{\partial} h(x)=\left\{z \mid(z,-1) \in \widehat{N}_{\text {epi }(h)}(x, h(x))\right\}$. The general (or limiting) subdifferential of $h$ at $x$ is given by

$$
\partial h(x):=\left\{z \mid \begin{array}{c}
\exists\left\{\left(x^{\nu}, z^{\nu}\right)\right\} \subset \operatorname{dom}(h) \times \mathbb{L}, \text { with } z^{\nu} \in \hat{\partial} h\left(x^{\nu}\right) \forall \nu, \\
\left(x^{\nu}, z^{\nu}\right) \rightarrow(x, z), \text { and } h\left(x^{\nu}\right) \rightarrow h(x)
\end{array}\right\},
$$


and the horizon subdifferential to $h$ at $x$ is given by

$$
\partial^{\infty} h(x):=\left\{\begin{array}{c}
z\left\{\begin{array}{c}
\left.\exists\left(x^{\nu}, z^{\nu}, \beta_{\nu}\right)\right\} \subset \operatorname{dom}(h) \times \mathbb{L} \times \mathbb{R}_{++}, \text {with } z^{\nu} \in \hat{\partial} h\left(x^{\nu}\right) \forall \nu \\
\beta_{\nu} \downarrow 0,\left(x^{\nu}, \beta_{\nu} z^{\nu}\right) \rightarrow(x, z), \text { and } h\left(x^{\nu}\right) \rightarrow h(x)
\end{array}\right.
\end{array}\right\} .
$$

The function $h$ is said to be subdifferentially regular at $x$ if $\partial h(x)=\hat{\partial} h(x)$ and $\partial^{\infty} h(x)=\hat{\partial} h(x)^{\infty}$. Subdifferential regularity allows the development of a rich subdifferential calculus.

If $h$ is differentiable at $x$, then, by [19, Exercise 8.8],

$$
\hat{\partial} h(x)=\partial h(x)=\{\nabla h(x)\} \quad \text { and } \quad \mathrm{d} h(x)(v)=\langle\nabla h(x), v\rangle .
$$

If $h$ is proper, convex, and lsc, then, by [19, Proposition 8.12], $h$ is subdifferentially regular at every $x \in \operatorname{dom}(h)$, in which case

$$
\mathrm{d} h(x)(v)=h^{\prime}(x ; v):=\lim _{t \downarrow 0}(h(x+t v)-h(x)) / t .
$$

Consequently, in this case, the subderivative corresponds to the usual notion of a directional derivative [19, page 257]. This notation is consistent with the usage in (10), where the function is assumed to be $\mathbb{R}$-differentiable.

\section{Polynomials}

The focus of study in 2, 9, 12, is polynomial root functions, a special class of which is polynomial root max functions (2). The goal is to apply the variational results for $\mathbf{f}$ in $[2,8,9$, 12, to $\mathfrak{f}$ when $\mathbf{f}$ is convexly generated. These functions depend on what will be called the active factor (see 3.2 and (47)). In the discussion of the application of the polynomial results to the characteristic polynomial, the integer $\tilde{n} \in \mathbb{N}$ is used to denote either the degree of the active factor of the characteristic polynomial (the nonderogatory case, 95 ) or the degree of the characteristic polynomial itself (the derogatory case, $\sqrt[6]{6}$ ).

3.1. Factorization spaces and their inner products on $\mathbb{P}^{\tilde{n}}[12$. Let $\leq$ denote the lexicographical order on $\mathbb{C}$, where, for $z_{s}:=x_{s}+\mathrm{i} y_{s}, x_{s}, y_{s} \in \mathbb{R}, s=1,2$, we have $z_{1} \leq z_{2}$ if and only if either $x_{1}<x_{2}$ or $\left(x_{1}=x_{2}\right.$ and $\left.y_{1} \leqslant y_{2}\right)$. Let $\mathbb{M}^{\tilde{n}} \subsetneq \mathbb{P}^{[\tilde{n}]}$ be the affine set of monic polynomials of degree $n$ with $\mathbb{M}^{0}:=\{1\}$. Given $\tilde{p} \in \mathbb{M}^{\tilde{n}}$, write

$$
\tilde{p}:=\prod_{j=1}^{m} e_{\left(n_{j}, \tilde{\lambda}_{j}\right)},
$$

where $\tilde{\lambda}_{1}, \ldots, \tilde{\lambda}_{m}$ are the distinct roots of $\tilde{p}$, ordered lexicographically with multiplicities $n_{1}, \ldots, n_{m}$, and the monomials $e_{\left(\ell, \tilde{\lambda}_{j}\right)} \in \mathbb{P}^{\tilde{n}}$ are defined by

$$
e_{\left(\ell, \lambda_{0}\right)}(\lambda):=\left(\lambda-\lambda_{0}\right)^{\ell}, \quad \text { for } \ell=0, \ldots, \tilde{n}, \text { and all } \lambda_{0} \in \mathbb{C} .
$$

Note that, for each fixed $\lambda_{0} \in \mathbb{C}$, the monomials (16) form a basis for the linear space $\mathbb{P}^{\tilde{n}}$. The factorization space $\mathbb{S}_{\tilde{p}}$ for $\tilde{p}$ is given by

$$
\mathbb{S}_{\tilde{p}}:=\mathbb{C} \times \mathbb{P}^{n_{1}-1} \times \mathbb{P}^{n_{2}-1} \times \cdots \times \mathbb{P}^{n_{m}-1},
$$

where the component indexing for elements of $\mathbb{S}_{\tilde{p}}$ starts at zero so that the $j$ th component is an element of $\mathbb{P}^{n_{j}-1}$. The spaces $\mathbb{P}^{\tilde{n}}$ and $\mathbb{S}_{\tilde{p}}$ are related through the mapping $F_{\tilde{p}}: \mathbb{S}_{\tilde{p}} \rightarrow \mathbb{P}^{\tilde{n}}$ given by

$$
F_{\tilde{p}}\left(q_{0}, q_{1}, q_{2}, \ldots, q_{m}\right):=\left(1+q_{0}\right) \prod_{j=1}^{m}\left(e_{\left(n_{j}, \tilde{\lambda}_{j}\right)}+q_{j}\right) .
$$


We have $F_{\tilde{p}}(0)=\prod_{j=1}^{m} e_{\left(n_{j}, \tilde{\lambda}_{j}\right)}=\tilde{p}$, and, since the factors in (15) are relatively prime, there exist neighborhoods $U$ of 0 in $\mathbb{S}_{\tilde{p}}$ and $V$ of $\tilde{p}$ in $\mathbb{P}^{\tilde{n}}$ such that $\left.F_{\tilde{p}}\right|_{U}: U \rightarrow$ $V$ is a diffeomorphism (over $\mathbb{C}$ ) [12, Lemma 1.4]. The $\mathbb{C}$-derivative $F_{\tilde{p}}^{\prime}(0): \mathbb{S}_{\tilde{p}} \rightarrow \mathbb{P}^{\tilde{n}}$ is invertible and given by

$$
F_{\tilde{p}}^{\prime}(0)\left(\omega_{0}, w_{1}, w_{2}, \ldots, w_{m}\right)=\omega_{0} \tilde{p}+\sum_{j=1}^{m} r_{j} w_{j}, \quad \text { where } r_{j}:=\tilde{p} / e_{\left(n_{j}, \tilde{\lambda}_{j}\right)} .
$$

For each $\lambda_{0} \in \mathbb{C}$, define the scalar Taylor maps $\tau_{\left(k, \lambda_{0}\right)}: \mathbb{P}^{\tilde{n}} \rightarrow \mathbb{C}$ by

$$
\tau_{\left(k, \lambda_{0}\right)}(q):=q^{(k)}\left(\lambda_{0}\right) / k !, \text { for } k=0,1,2, \ldots, \tilde{n},
$$

where $q^{(\ell)}$ is the $\ell$ th derivative of $q$. Each mapping $\tau_{\left(k, \lambda_{0}\right)}$ takes a polynomial to its $k$ th Taylor coefficient at $\lambda_{0}$. Note that the mapping $\tilde{\tau}_{k}(q, \lambda):=\tau_{(k, \lambda)}(q)$ is continuous in $q$ and $\lambda\left[12\right.$. Define the $\mathbb{C}$-linear isomorphism $\mathcal{T}_{\tilde{p}}: \mathbb{S}_{\tilde{p}} \rightarrow \mathbb{C}^{\tilde{n}+1}$ by

$$
\begin{aligned}
\mathcal{T}_{\tilde{p}}(u):=\left[\mu_{0},\left(\tau_{\left(n_{1}-1, \tilde{\lambda}_{1}\right)}\left(u_{1}\right), \ldots, \tau_{\left(0, \tilde{\lambda}_{1}\right)}\left(u_{1}\right)\right), \ldots,\right. \\
\left.\left(\tau_{\left(n_{m}-1, \tilde{\lambda}_{m}\right)}\left(u_{m}\right), \ldots, \tau_{\left(0, \tilde{\lambda}_{m}\right)}\left(u_{m}\right)\right)\right]^{T} \\
=\left[\mu_{0},\left(\mu_{11}, \ldots, \mu_{1 n_{j}}\right), \ldots,\left(\mu_{m 1}, \ldots, \mu_{m n_{m}}\right)\right]^{T},
\end{aligned}
$$

where

$$
u:=\left(\mu_{0}, u_{1}, u_{2}, \ldots, u_{m}\right) \in \mathbb{S}_{\tilde{p}}, u_{j}:=\sum_{s=1}^{n_{j}} \mu_{j s} e_{\left(n_{j}-s, \tilde{\lambda}_{j}\right)}, \text { and } \mu_{0}, \mu_{j s} \in \mathbb{C},
$$

for all $s \in\left\{1,2, \ldots n_{j}\right\}$ and $j \in\{1,2, \ldots m\}$.

By Lemma 2, $\mathcal{T}_{\tilde{p}}$ induces an inner product on $\mathbb{S}_{\tilde{p}}$ by setting

$$
\langle u, w\rangle_{\mathbb{S}_{\tilde{p}}}^{\mathfrak{c}}:=\left\langle\mathcal{T}_{\tilde{p}}(u), \mathcal{T}_{\tilde{p}}(w)\right\rangle_{\mathbb{C}^{\tilde{n}+1}}^{\mathfrak{c}} \text {, for all } u, w \in \mathbb{S}_{\tilde{p}},
$$

where $u$ is given in (21),

$$
\begin{gathered}
w=\left(\omega_{0}, w_{1}, w_{2}, \ldots, w_{m}\right), \quad \text { with } \\
w_{j}=\sum_{s=1}^{n_{j}} \omega_{j s} e_{\left(n_{j}-s, \tilde{\lambda}_{j}\right)} \text { for } j \in\{1, \ldots, m\} \text { and } \\
\omega_{0}, \omega_{j s} \in \mathbb{C} \text { for } s \in\left\{1, \ldots, n_{j}\right\}, j \in\{1, \ldots, m\} .
\end{gathered}
$$

Moreover, again by Lemma 2 ,

$$
\mathcal{T}_{\tilde{p}}^{\star}=\mathcal{T}_{\tilde{p}}^{-1}
$$

with respect to the inner products $\langle\cdot, \cdot\rangle_{\mathbb{S}_{\tilde{p}}}^{\mathfrak{c}}$ and $\langle\cdot, \cdot\rangle_{\mathbb{C}^{\tilde{n}+1}}^{\mathfrak{c}}$.

We are now ready to construct an inner product on $\mathbb{P}^{\tilde{n}}$ relative to $\tilde{p}$. Recall that $F_{\tilde{p}}^{\prime}(0): \mathbb{S}_{\tilde{p}} \rightarrow \mathbb{P}^{\tilde{n}}$ given in (18) is a $\mathbb{C}$-linear isomorphism. So for every $z, v \in \mathbb{P}^{\tilde{n}}$, there exists $u, w \in \mathbb{S}_{\tilde{p}}$, having representations (21) and (23), such that

$$
z=F_{\tilde{p}}^{\prime}(0) u \quad \text { and } \quad v=F_{\tilde{p}}^{\prime}(0) w .
$$

By Lemma 2, $F_{\tilde{p}}^{\prime}(0)^{-1}$ induces an inner product $\langle\cdot, \cdot\rangle_{(\mathbb{P} \tilde{n}, \tilde{p})}^{\mathfrak{c}}$ on $\mathbb{P}^{\tilde{n}}$ based on the inner product $\langle\cdot, \cdot\rangle_{\mathbb{S}_{\tilde{p}}}^{\mathfrak{c}}$ by setting

$$
\begin{aligned}
\langle z, v\rangle_{(\mathbb{P} \tilde{n}, \tilde{p})}^{\mathfrak{c}} & :=\left\langle F_{\tilde{p}}^{\prime}(0)^{-1} z, F_{\tilde{p}}^{\prime}(0)^{-1} v\right\rangle_{\mathbb{S}_{\tilde{p}}}^{\mathfrak{c}}=\langle u, w\rangle_{\mathbb{S}_{\tilde{p}}}^{\mathfrak{c}} \\
& =\left\langle\left(\mu_{0}, u_{1}, u_{2}, \ldots, u_{m}\right),\left(\omega_{0}, w_{1}, w_{2}, \ldots, w_{m}\right)\right\rangle_{\mathbb{S}_{\tilde{p}}}^{\mathfrak{c}} \\
& =\bar{\mu}_{0} \omega_{0}+\sum_{j=1}^{m} \sum_{s=1}^{n_{j}} \bar{\mu}_{j s} \omega_{j s},
\end{aligned}
$$

where $z$ and $v$ are given in (24), and $u$ and $w$ are given in (21) and (23), respectively. With respect to these inner products, (25) (or Lemma 21) gives

$$
\left(F_{\tilde{p}}^{\prime}(0)\right)^{\star}=F_{\tilde{p}}^{\prime}(0)^{-1} .
$$


3.2. Active roots and active indices. Let $p \in \mathbb{P}^{[\tilde{n}]}$ and denote by $\Xi_{p}:=\left\{\lambda_{1}, \ldots, \lambda_{m}\right\}$ the distinct roots of $p$, ordered lexicographically. The set of active roots of $\mathbf{f}$ at $p$ is given by

$$
\mathcal{A}_{\mathbf{f}}(p):=\left\{\lambda_{j} \in \Xi_{p} \mid f\left(\lambda_{j}\right)=\mathbf{f}(p)\right\} .
$$

If $\lambda_{j} \in \Xi_{p} \backslash \mathcal{A}_{\mathbf{f}}(p)$, then $f\left(\lambda_{j}\right)<\mathbf{f}(p)$, and we say that $\lambda_{j}$ is inactive. The set of active indices of $\mathbf{f}$ at $p$ is given by

$$
\mathcal{I}_{\mathbf{f}}(p):=\left\{j \in\{1, \ldots, m\} \mid \lambda_{j} \in \mathcal{A}_{\mathbf{f}}(p)\right\} .
$$

3.3. The subdifferential and subderivative for polynomial root max functions. Again assume that $f: \mathbb{C} \rightarrow \overline{\mathbb{R}}$ is proper, convex, and lsc. As discussed at the end of Section 2.3. $f$ is subdifferentially regular and so (14) holds.

Theorem 3. 2, Proposition 5.5, Theorem 5.3 and Theorem 6.2] Let $\tilde{p} \in \mathbb{P}^{\tilde{n}} \cap$ $\operatorname{dom}(\mathbf{f})$ have degree $\tilde{n}$ with decomposition (15). Assume that $f$ satisfies either (3) or (44) at $\tilde{\lambda}_{j}$ for $j \in \mathcal{I}_{\mathbf{f}}(\tilde{p})$, and that

$$
\partial f\left(\tilde{\lambda}_{j}\right) \neq\{0\} \quad \forall j \in \mathcal{I}_{\mathbf{f}}(\tilde{p}) .
$$

Given $j \in \mathcal{I}_{\mathbf{f}}(\tilde{p})$, define

$$
\mathcal{Q}\left(\tilde{\lambda}_{j}\right):=-\operatorname{cone}\left(\partial f\left(\tilde{\lambda}_{j}\right)^{2}\right)+\mathrm{i}\left(\operatorname{rspan}\left(\partial f\left(\tilde{\lambda}_{j}\right)^{2}\right)\right) \subset \mathbb{C},
$$

where $\partial f\left(\tilde{\lambda}_{j}\right)^{2}:=\left\{g^{2} \mid g \in \partial f\left(\tilde{\lambda}_{j}\right)\right\}$ and, for $S \subset \mathbb{C}$,

$$
\text { cone }(S):=\left\{\tau \zeta \mid \tau \in \mathbb{R}_{+}, \zeta \in S\right\} \text {. }
$$

For $\tilde{\lambda}_{j} \in \mathcal{A}_{\mathbf{f}}(\tilde{p})$ at which (3) holds, define

$$
\mathcal{D}\left(n_{j}, \tilde{\lambda}_{j}\right):=\left\{\theta \mid\left\langle\theta,\left(\nabla f\left(\tilde{\lambda}_{j}\right)\right)^{2}\right\rangle_{\mathbb{C}} \leqslant\left\langle\mathrm{i} \nabla f\left(\tilde{\lambda}_{j}\right), \nabla^{2} f\left(\tilde{\lambda}_{j}\right)\left(\mathrm{i} \nabla f\left(\tilde{\lambda}_{j}\right)\right)\right\rangle_{\mathbb{C}} / n_{j}\right\} \subset \mathbb{C} .
$$

Next, set

$$
D_{\tilde{p}}:=\operatorname{conv}\left(\{0\} \times \underset{j=1}{\mathrm{~m}} \Gamma\left(n_{j}, \tilde{\lambda}_{j}\right)\right) \subset \mathbb{C}^{\tilde{n}+1},
$$

where, for $j \notin \mathcal{I}_{\mathbf{f}}(\tilde{p}), \Gamma\left(n_{j}, \tilde{\lambda}_{j}\right):=\{0\} \subset \mathbb{C}^{n_{j}}$ and, for $j \in \mathcal{I}_{\mathbf{f}}(\tilde{p})$,

$$
\Gamma\left(n_{j}, \tilde{\lambda}_{j}\right):=\left\{\begin{array}{ll}
\left(-\partial f\left(\tilde{\lambda}_{j}\right) / n_{j}\right) \times \mathcal{D}\left(n_{j}, \tilde{\lambda}_{j}\right) \times \mathbb{C}^{n_{j}-2} & \text { if (3) holds at } \tilde{\lambda}_{j}, \\
\left(-\partial f\left(\tilde{\lambda}_{j}\right) / n_{j}\right) \times \mathcal{Q}\left(\tilde{\lambda}_{j}\right) \times \mathbb{C}^{n_{j}-2} & \text { if (4) holds at } \tilde{\lambda}_{j}
\end{array} \subset \mathbb{C}^{n_{j}} .\right.
$$

Then

$$
D_{\tilde{p}}^{\infty}=\{0\} \times \underset{j=1}{m} \Gamma\left(n_{j}, \tilde{\lambda}_{j}\right)^{\infty} \quad \text { with } \quad \Gamma\left(n_{j}, \tilde{\lambda}_{j}\right)^{\infty}=\{0\} \times \mathcal{Q}\left(\tilde{\lambda}_{j}\right) \times \mathbb{C}^{n_{j}-1},
$$

and, with respect to the inner product $\langle\cdot, \cdot\rangle_{(\mathbb{P} \tilde{n}, \tilde{p})}$ given in (26),

$$
\hat{\partial} \mathbf{f}(\tilde{p})=F_{\tilde{p}}^{\prime}(0) \circ \mathcal{T}_{\tilde{p}}^{-1}\left(D_{\tilde{p}}\right) \quad \text { and } \quad \hat{\partial} \mathbf{f}(\tilde{p})^{\infty}=F_{\tilde{p}}^{\prime}(0) \circ \mathcal{T}_{\tilde{p}}^{-1}\left(D_{\tilde{p}}^{\infty}\right),
$$

where $F_{\tilde{p}}^{\prime}(0)$ and $\mathcal{T}_{\tilde{p}}$ are given in (18) and (20), respectively.

If $f$ satisfies (3) for all $\tilde{\lambda}_{j} \in \mathcal{A}_{\mathbf{f}}(\tilde{p})$, then $\mathbf{f}$ is subdifferentially regular at $\tilde{p}$ and (30) gives the general and horizon subdifferentials, respectively, for $\mathbf{f}$ at $\tilde{p}$. 
Theorem 4. 2, Theorem 3.3] Let $f$ and $\tilde{p}$ satisfy the hypotheses of Theorem 3 , Let $v=F_{\tilde{p}}^{\prime}(0)\left(\omega_{0}, w_{1}, \ldots, w_{m}\right)$, where $w_{j}=\sum_{s=1}^{n_{j}} \omega_{j s} e_{\left(n_{j}-s, \tilde{\lambda}_{j}\right)} \in \mathbb{P}^{n_{j}-1}$ for all $j \in$ $\{1, \ldots m\}$, with $\omega_{j s} \in \mathbb{C}$, for $s \in\left\{1, \ldots, n_{j}\right\}$. If $v$ satisfies

$$
\begin{array}{ll}
0=\left\langle g, \sqrt{-\omega_{j 2}}\right\rangle_{\mathbb{C}} & \text { for all } j \in \mathcal{I}_{\mathbf{f}}(\tilde{p}) \text { and } g \in \partial f\left(\tilde{\lambda}_{j}\right), \text { and } \\
0=\omega_{j s} & \text { for all } s \in\left\{3, \ldots, n_{j}\right\} \text { and } j \in \mathcal{I}_{\mathbf{f}}(\tilde{p}),
\end{array}
$$

then

$$
\mathrm{d} \mathbf{f}(\tilde{p})(v)=\max _{j \in \mathcal{I}_{\mathbf{f}}(\tilde{p})}\left\{\left(f^{\prime}\left(\tilde{\lambda}_{j} ;-\omega_{j 1}\right)+\kappa_{j}\right\}\right.
$$

where, for $j \in \mathcal{I}_{\mathbf{f}}(\tilde{p})$,

$$
\kappa_{j}:= \begin{cases}\left.f^{\prime \prime}\left(\tilde{\lambda}_{j} ; \sqrt{-\omega_{j 2}}, \sqrt{-\omega_{j 2}}\right)\right) / n_{j} & \text { if (3) holds at } \tilde{\lambda}_{j}, \\ 0 & \text { if (4) holds at } \tilde{\lambda}_{j} ;\end{cases}
$$

otherwise, $\mathrm{d} \mathbf{f}(\tilde{p})(v)=+\infty$. In addition, $\mathrm{d} \mathbf{f}(\tilde{p})$ is proper, lsc, and sublinear.

\section{The Derivative of the Nonderogatory Factor}

We now recall Arnold form [1] and its application in [5] for describing the local variational behavior of the Jordan form.

\subsection{Arnold Form. Let}

$$
\widetilde{\Xi}:=\left\{\tilde{\lambda}_{1}, \ldots, \tilde{\lambda}_{m}\right\}
$$

be a subset of the distinct eigenvalues of $\tilde{X} \in \mathbb{C}^{n \times n}$. The Jordan structure of $\tilde{X}$ relative to these eigenvalues is given by

$$
J:=\widetilde{P} \widetilde{X} \widetilde{P}^{-1}=\operatorname{Diag}\left(\widetilde{B}, J_{1}, \ldots, J_{m}\right), \text { where } J_{j}:=\operatorname{Diag}\left(J_{j}^{(1)}, \ldots, J_{j}^{\left(q_{j}\right)}\right)
$$

and $J_{j}^{(k)}$ is an $m_{j k} \times m_{j k}$ Jordan block

$$
J_{j}^{(k)}:=\tilde{\lambda}_{j} I_{m_{j k}}+N_{j k}, \quad k=1, \ldots, q_{j}, j=1, \ldots, m,
$$

where $N_{j k} \in \mathbb{C}^{m_{j k} \times m_{j k}}$ is the nilpotent matrix given by ones on the superdiagonal and zeros elsewhere, and $I_{m_{j k}} \in \mathbb{C}^{m_{j k} \times m_{j k}}$ is the identity matrix. With this notation, $q_{j}$ is the geometric multiplicity of the eigenvalue $\tilde{\lambda}_{j}$. The algebraic multiplicity of $\tilde{\lambda}_{j}$ is $n_{j}:=\sum_{k=1}^{q_{j}} m_{j k}$. The size of the largest Jordan block for an eigenvalue is

$$
m_{j}:=\max _{k=1, \ldots, q_{j}} m_{j k} .
$$

Set $\tilde{n}:=\sum_{j=1}^{m} n_{j}$ and $n_{0}:=n-\tilde{n}$. The matrix $\widetilde{B} \in \mathbb{C}^{n_{0} \times n_{0}}$ (possibly of size 0 ) corresponds to the eigenvalues not included in $\widetilde{\Xi}$. The eigenvalue $\tilde{\lambda}_{j}$ is nonderogatory if $q_{j}=1$ (equivalently $m_{j}=n_{j}$ ). The matrix $\tilde{X}$ is nonderogatory if all of its eigenvalues are nonderogatory.

Theorem 5 (Arnold Form). [5, Theorems 2.2, 2.7] Suppose that all eigenvalues in $\widetilde{\Xi}$ are nonderogatory. We suppress the index $k$ since $q_{j}=1$. Then there exists a 
neighborhood $\Omega$ of $\tilde{X} \in \mathbb{C}^{n \times n}$ and smooth maps $P: \Omega \rightarrow \mathbb{C}^{n \times n}, B: \Omega \rightarrow \mathbb{C}^{n_{0} \times n_{0}}$ and, for $j \in\{1, \ldots, m\}$ and $s \in\left\{0,1, \ldots, n_{j}-1\right\}, \lambda_{j s}: \Omega \rightarrow \mathbb{C}$ such that

$$
\begin{aligned}
P(X) X P(X)^{-1} & =\operatorname{Diag}(B(X), 0, \ldots, 0)+\sum_{j=1}^{m} \check{J}_{j}(X) \in \mathbb{C}^{n \times n}, \\
\lambda_{j s}(\tilde{X}) & =0, \quad s=0,1, \ldots, n_{j}-1, \\
P(\widetilde{X}) & =\widetilde{P}, \quad B(\tilde{X})=\widetilde{B}, \quad \text { and } \\
P(\widetilde{X}) \widetilde{X} P(\widetilde{X})^{-1} & =\operatorname{Diag}\left(B(\widetilde{X}), J_{1}, \ldots, J_{m}\right),
\end{aligned}
$$

where

$$
\begin{aligned}
\check{J}_{j}(X) & :=\tilde{\lambda}_{j} J_{j 0}+J_{j 1}+\sum_{s=0}^{n_{j}-1} \lambda_{j s}(X) J_{j s}^{*}, \\
J_{j s} & :=\operatorname{Diag}\left(0, \ldots, 0, N_{j}^{s}, 0, \ldots, 0\right), \quad \text { and } \\
J_{j 0} & :=\operatorname{Diag}\left(0, \ldots, 0, I_{n_{j}}, 0, \ldots, 0\right),
\end{aligned}
$$

with $N_{j}^{s}$ and $I_{n_{j}}$ in the $\tilde{\lambda}_{j}$ diagonal block (see (34)). Finally, the functions $\lambda_{j s}$ are uniquely defined on $\Omega$, though the maps $P$ and $B$ are not unique.

Remark 6. Theorem 5 illustrates a fundamental difference between the symmetric and nonsymmetric cases. In the symmetric case, the matrices are unitarily diagonalizable so there are no nilpotent matrices $N_{j}$ and the mappings $\lambda_{j s}$ reduce to the eigenvalue mapping $\lambda_{j}$. In this case, a seminal result due to Lewis [16. Theorem 6] shows that the variational properties depend on the eigenvalues. On the other hand, in the nonsymmetric case they depend on the entire family of functions $\lambda_{j s}$.

Lemma 7. 5, Lemma 2.12]. Assume the hypotheses of Theorem 5. Then the gradients (see (8) ) of the functions $\lambda_{j s}: \mathbb{C}^{n \times n} \rightarrow \mathbb{C}$ are given by $\nabla \lambda_{j s}(\tilde{X})=$ $\left(n_{j}-s\right)^{-1} \widetilde{P}^{*} J_{j s}^{*} \widetilde{P}^{-*}$, and

$$
\left(\lambda_{j s}^{\prime}(\tilde{X})\right)^{\star} \zeta=\zeta\left(n_{j}-s\right)^{-1} \widetilde{P}^{*} J_{j s}^{*} \widetilde{P}^{-*} \quad \forall \zeta \in \mathbb{C}
$$

with respect to the inner product $\langle\cdot, \cdot\rangle_{\mathbb{C}^{n \times n}}^{\mathfrak{c}}($ see (9) $)$.

4.2. The derivative of the nonderogatory characteristic factors. The local factorization of $X$ on $\Omega$ described in Theorem 5 can be used to describe a local factorization of the characteristic polynomial $\operatorname{det}(\lambda I-X)$ near $\tilde{X}$. The following technical lemma allows us to represent the coefficients of the factors of the characteristic polynomial in terms of the functions $\lambda_{j s}$ in Theorem 5.

Lemma 8. Let $J \in \mathbb{C}^{n \times n}$ be a Jordan matrix having ones on the superdiagonal and zeros elsewhere, $\xi \in \mathbb{C}$, and $\lambda=\left(\lambda_{0}, \ldots, \lambda_{n-1}\right) \in \mathbb{C}^{n}$. Consider the matrix

$$
\xi I_{n}-J-\sum_{s=0}^{n-1} \lambda_{s}\left(J^{*}\right)^{s}=\xi I_{n}-\left[\begin{array}{ccccc}
\lambda_{0} & 1 & 0 & \cdots & 0 \\
\lambda_{1} & \lambda_{0} & 1 & \cdots & 0 \\
\vdots & \vdots & \vdots & \ddots & 1 \\
\lambda_{n-1} & \lambda_{n-2} & \lambda_{n-3} & \cdots & \lambda_{0}
\end{array}\right] .
$$

Then

$$
\operatorname{det}\left(\xi I_{n}-J-\sum_{s=0}^{n-1} \lambda_{s}\left(J^{*}\right)^{s}\right)=\xi^{n}-\sum_{s=0}^{n-1}(n-s) \lambda_{s} \xi^{n-s-1}+o(\lambda)
$$

where $J^{0}:=I_{n}$ 
Proof. First consider matrices of the form

$$
A_{s}:=\left[\begin{array}{ccccc}
a_{0} & -1 & 0 & \cdots & 0 \\
a_{1} & a_{0} & -1 & \cdots & 0 \\
a_{2} & a_{1} & a_{0} & \cdots & 0 \\
\vdots & \vdots & \vdots & \ddots & -1 \\
a_{s-1} & a_{s-2} & a_{s-3} & \cdots & a_{0}
\end{array}\right] \in \mathbb{C}^{s \times s},
$$

for $s=1, \ldots, n$, where $a_{0}, a_{1}, \ldots, a_{s-1} \in \mathbb{C}$ so that for $s=1, \ldots, n-1, A_{s}$ is the lower right (or upper left) $s \times s$ block of $A_{s+1}$. Note that $\operatorname{det}\left(A_{n}\right)$ equals

$$
a_{n-1}+a_{n-2} \operatorname{det}\left(A_{1}\right)+a_{n-3} \operatorname{det}\left(A_{2}\right)+\cdots+a_{1} \operatorname{det}\left(A_{n-2}\right)+a_{0} \operatorname{det}\left(A_{n-1}\right) .
$$

To see this, expand the determinant on the first column of $A_{n}$ and observe that when the $s$ th row and first column is deleted from $A_{n}$, the resulting $(n-1) \times(n-1)$ matrix is lower block triangular with only two diagonal blocks, where the upper diagonal block is an $(s-1) \times(s-1)$ lower triangular matrix with -1 in every diagonal entry and the lower diagonal $(n-s) \times(n-s)$ block equals $A_{n-s}$.

To establish the lemma, we need to find $\operatorname{det}\left(A_{n}\right)$ with $a_{0}=\xi-\lambda_{0}$ and $a_{s}=-\lambda_{s}$ for $s=1, \ldots, n-1$. For $n=1, A_{1}=\left(\xi-\lambda_{0}\right)$. For $n=2$,

$$
\operatorname{det}\left[\begin{array}{rr}
\left(\xi-\lambda_{0}\right) & -1 \\
-\lambda_{1} & \left(\xi-\lambda_{0}\right)
\end{array}\right]=\left(\xi-\lambda_{0}\right)^{2}-\lambda_{1}=\xi^{2}-2 \lambda_{0} \xi-\lambda_{1}+o(\lambda) .
$$

Suppose (37) holds for $s=1, \ldots, n-1$. This together with (38) implies

$$
\begin{aligned}
\operatorname{det}\left(A_{n}\right)= & -\lambda_{n-1}-\lambda_{n-2}\left(\xi-\lambda_{0}\right)-\lambda_{n-3}\left(\xi^{2}-2 \lambda_{0} \xi-\lambda_{1}\right)-\cdots \\
& -\lambda_{1}\left(\xi^{n-2}-\sum_{s=0}^{(n-2)-1}((n-2)-s) \lambda_{s} \xi^{(n-2)-s-1}\right) \\
& +\left(\xi-\lambda_{0}\right)\left(\xi^{n-1}-\sum_{s=0}^{(n-1)-1}((n-1)-s) \lambda_{s} \xi^{(n-1)-s-1}\right)+o(\lambda) .
\end{aligned}
$$

Collecting like powers of $\xi$ establishes the result:

$$
\begin{aligned}
\operatorname{det}\left(A_{n}\right) & =\xi^{n}-\lambda_{0} \xi^{n-1}-\sum_{s=0}^{(n-1)-1}((n-1)-s) \lambda_{s} \xi^{n-s-1}-\sum_{s=1}^{n-1} \lambda_{s} \xi^{n-s-1}+o(\lambda) \\
& =\xi^{n}-\lambda_{0} \xi^{n-1}-(n-1) \lambda_{0} \xi^{n-1}-\left(\sum_{s=1}^{(n-1)-1}(n-s) \lambda_{s} \xi^{n-s-1}\right)-\lambda_{n-1}+o(\lambda), \\
& =\xi^{n}-n \lambda_{0} \xi^{n-1}-\left(\sum_{s=1}^{(n-1)-1}(n-s) \lambda_{s} \xi^{n-s-1}\right)-\lambda_{n-1}+o(\lambda), \\
& =\xi^{n}-\sum_{s=0}^{n-1}(n-s) \lambda_{s} \xi^{n-s-1}+o(\lambda) .
\end{aligned}
$$

Lemma 9. Assume the hypotheses of Theorem 5. Let $j \in\{1, \ldots, m\}$ and define $g_{j}: \Omega \rightarrow \mathbb{P}^{n_{j}-1}$ by

$$
g_{j}(X):=\Phi_{n_{j}}\left(\widehat{J}_{j}(X)\right)-e_{\left(n_{j}, \tilde{\lambda}_{j}\right)},
$$

where $\Phi_{n_{j}}$ and $e_{\left(n_{j}, \tilde{\lambda}_{j}\right)}$ are defined in (5) and (16), respectively, and

$$
\widehat{J}_{j}(X):=\tilde{\lambda}_{j} I_{n_{j}}+J_{j}+\sum_{s=0}^{n_{j}-1} \lambda_{j s}(X)\left(J_{j}^{s}\right)^{*} .
$$

Then

$$
g_{j}(X)=-\sum_{s=0}^{n_{j}-1}\left(n_{j}-s\right) \lambda_{j s}(X) e_{\left(n_{j}-s-1, \tilde{\lambda}_{j}\right)}+o\left(\lambda_{j 0}(X), \ldots, \lambda_{j\left(n_{j}-1\right)}(X)\right) .
$$

Moreover, $g_{j}(\tilde{X})=0$, and, with respect to the inner products

$$
\langle\cdot, \cdot\rangle_{\left[n_{j}-1, \tilde{\lambda}_{j}\right]}^{\mathfrak{c}}:=\sum_{s=0}^{n_{j}-1}\left\langle\tau_{\left(n_{j}-s-1, \tilde{\lambda}_{j}\right)}(\cdot), \tau_{\left(n_{j}-s-1, \tilde{\lambda}_{j}\right)}(\cdot)\right\rangle_{\mathbb{C}}^{\mathfrak{c}}
$$


on $\mathbb{P}^{n_{j}-1}$ and $\langle\cdot, \cdot\rangle_{\mathbb{C}^{n \times n}}^{\mathfrak{c}}$ on $\mathbb{C}^{n \times n}$,

$$
\left(g_{j}^{\prime}(\widetilde{X})\right)^{\star}=-\sum_{s=0}^{n_{j}-1} \widetilde{P}^{*} J_{j s}^{*} \widetilde{P}^{-*} \tau_{\left(n_{j}-s-1, \tilde{\lambda}_{j}\right)},
$$

where the mapping $\tau_{\left(k, \lambda_{0}\right)}$ is defined in (19). In particular, $\left(g_{j}^{\prime}(\tilde{X})\right)^{\star}$ is injective.

Proof. Let $j \in\{1, \ldots, m\}$ and $g_{j}$ be as given in (39). Combining Theorem 5 with Lemma 8 where $\xi:=\left(\lambda-\tilde{\lambda}_{j}\right), J:=J_{j}$ and $n:=n_{j}$ gives (41). Since $\lambda_{j s}(\tilde{X})=0$ for $s=0,1, \ldots, n_{j}-1$, we have $g_{j}(\tilde{X})=0$ and

$$
g_{j}^{\prime}(X)=-\sum_{s=0}^{n_{j}-1}\left(n_{j}-s\right) e_{\left(n_{j}-s-1, \tilde{\lambda}_{j}\right)} \lambda_{j s}^{\prime}(X) .
$$

Let $M \in \mathbb{C}^{n \times n}$ and set $h:=\sum_{k=0}^{n_{j}-1} c_{k} e_{\left(n_{j}-k-1, \tilde{\lambda}_{j}\right)} \in \mathbb{P}^{n_{j}-1}$. Then

$$
\begin{aligned}
& \left\langle\left(g_{j}^{\prime}(\tilde{X})\right)^{\star} h, M\right\rangle_{\mathbb{C}^{n \times n}}^{\mathfrak{c}} \\
& =\left\langle h, g_{j}^{\prime}(\tilde{X}) M\right\rangle_{\left[n_{j}-1, \tilde{\lambda}_{j}\right]}^{\mathfrak{c}} \\
& =-\left\langle\sum_{k=0}^{n_{j}-1} c_{k} e_{\left(n_{j}-k-1, \tilde{\lambda}_{j}\right)},\left(\sum_{s=0}^{n_{j}-1}\left(n_{j}-s\right) e_{\left(n_{j}-s-1, \tilde{\lambda}_{j}\right)} \lambda_{j s}^{\prime}(\tilde{X})\right) M\right\rangle_{\left[n_{j}-1, \tilde{\lambda}_{j}\right]}^{\mathfrak{c}} \\
& =-\sum_{s=0}^{n_{j}-1}\left\langle c_{s},\left(n_{j}-s\right) \lambda_{j s}^{\prime}(\tilde{X}) M\right\rangle_{\mathbb{C}}^{\mathfrak{c}} \\
& =-\sum_{s=0}^{n_{j}-1}\left\langle\left(\lambda_{j s}^{\prime}(\tilde{X})\right)^{\star} c_{s},\left(n_{j}-s\right) M\right\rangle_{\mathbb{C}^{n \times n}}^{\mathfrak{c}} \quad \text { (by Lemma 7) } \\
& =-\sum_{s=0}^{n_{j}-1}\left\langle\left(n_{j}-s\right)^{-1} \widetilde{P}^{*} J_{j s}^{*} \widetilde{P}^{-*} c_{s},\left(n_{j}-s\right) M\right\rangle_{\mathbb{C}^{n \times n}}^{\mathfrak{c}} \quad \\
& =-\sum_{s=0}^{n_{j}-1}\left\langle\widetilde{P}^{*} J_{j s}^{*} \widetilde{P}^{-*} c_{s}, M\right\rangle_{\mathbb{C}^{n \times n}}^{\mathfrak{c}} \\
& =\left\langle\left(-\sum_{s=0}^{n_{j}-1} \widetilde{P}^{*} J_{j s}^{*} \widetilde{P}^{-*} \tau_{\left(n_{j}-s-1, \tilde{\lambda}_{j}\right)}\right) h, M\right\rangle_{\mathbb{C}^{n \times n}}^{\mathfrak{c}},
\end{aligned}
$$

which proves (42). Moreover, the matrices $\left\{\widetilde{P}^{*} J_{j s}^{*} \widetilde{P}^{-*}\right\}_{s=0}^{n_{j}-1}$ are linearly independent on $\mathbb{C}^{n \times n}($ over $\mathbb{C}$ or $\mathbb{R})$ and $\tau_{\left(n_{j}-s-1, \tilde{\lambda}_{j}\right)}(h)=0$ for all $s=0, \ldots, n_{j}-1$ only if $h=0$. Hence $\left(g_{j}^{\prime}(\tilde{X})\right)^{\star} h=0$ only if $h=0$, implying $\left(g_{j}^{\prime}(\tilde{X})\right)^{\star}$ is injective.

We now combine the functions $g_{j}$ into a single function $G: \mathbb{C} \times \Omega \rightarrow \mathbb{S}_{\tilde{p}}$, where $\tilde{p} \in \mathbb{P}^{\tilde{n}}$ is the polynomial in (15) with $\widetilde{\Xi}:=\left\{\tilde{\lambda}_{1}, \ldots, \tilde{\lambda}_{m}\right\}$ as in (33):

$$
G(\zeta, X):=\left(\zeta, g_{1}(X), \ldots, g_{m}(X)\right) .
$$

Note that the mapping $G$ depends on $\tilde{X}$, is $\mathbb{C}$-linear (the identity on $\mathbb{C}$ ) in its first component, and satisfies

$$
G(0, \tilde{X})=(0,0, \ldots, 0)=0
$$

Theorem 10 (Nonderogatory Characteristic Factor Derivatives). Let the hypotheses of Theorem 5 hold. Let $G:\{\zeta:|\zeta|<1\} \times \Omega \rightarrow \mathbb{S}_{\tilde{p}}$ be as in (43).

Let $R: \mathbb{C}^{\tilde{n}+1} \rightarrow \mathbb{C} \times \mathbb{C}^{n \times n}$ be the $\mathbb{C}$-linear transformation given by

$$
R(v):=\left(v_{0},-\sum_{j=1}^{m} \sum_{s=0}^{n_{j}-1} v_{j s} \widetilde{P}^{*} J_{j s}^{*} \widetilde{P}^{-*}\right),
$$

for all $v:=\left(v_{0}, v_{10}, \ldots, v_{1\left(n_{1}-1\right)}, \ldots, v_{m 0}, \ldots, v_{m\left(n_{m}-1\right)}\right) \in \mathbb{C}^{\tilde{n}+1}$. Then

$$
\left(G^{\prime}(\zeta, \tilde{X})\right)^{\star}=R \circ \mathcal{T}_{\tilde{p}}
$$


with respect to the inner products $\langle\cdot, \cdot\rangle_{\mathbb{S}_{\tilde{p}}}^{\mathfrak{c}}($ see (22) $)$ and $\langle(\zeta, X),(\omega, Y)\rangle^{\mathfrak{c}}:=\langle\zeta, \omega\rangle_{\mathbb{C}}^{\mathfrak{c}}+$ $\langle X, Y\rangle_{\mathbb{C}^{n \times n}}^{\mathfrak{c}}$ on $\mathbb{C} \times \mathbb{C}^{n \times n}$, where $\mathcal{T}_{\tilde{p}}$ is defined in (20). In addition, $\left(G^{\prime}(\zeta, \tilde{X})\right)^{\star}$ is injective.

Proof. The representation (46) follows immediately from Lemma 9 . Injectivity follows from the linear independence of the matrices $J_{j s} \in \mathbb{C}^{n \times n}$ for $s=0, \ldots, n_{j}-1$ and $j=1, \ldots, m$, and the fact that $\mathcal{T}_{\tilde{p}}$ is a $\mathbb{C}$-linear isomorphism.

\section{Matrices: Chain RUle For the NONDEROGatory CASE}

Let us now suppose that the eigenvalues in $\widetilde{\Xi}$ (see $\underline{(33)}$ ) ) are the active roots (see (28)) of the characteristic polynomial $\Phi_{n}(\tilde{X})$. We call $\widetilde{\Xi}$ the set of active eigenvalues of $\mathfrak{f}$ at $\tilde{X}$, denoted by $\mathcal{A}_{\mathfrak{f}}(\tilde{X})$. The corresponding active indices are denoted by $\mathcal{I}_{\mathfrak{f}}(\tilde{X})$. In (15), $\tilde{p}$ is called the active factor of the characteristic polynomial

$$
\Phi_{n}(\tilde{X})=\Phi_{\tilde{n}}(\hat{J}(\tilde{X})) \Phi_{n_{0}}(B(\tilde{X}))=\tilde{p} \Phi_{n_{0}}(B(\tilde{X})),
$$

where

$$
\widehat{J}(X):=\operatorname{Diag}\left(\widehat{J}_{1}(X), \ldots, \widehat{J}_{m}(X)\right),
$$

with $\widehat{J}_{j}$ defined in (40). If all active eigenvalues are nonderogatory, we have

$$
\mathfrak{f}(X)=\left(\mathbf{f} \circ F_{\tilde{p}} \circ G\right)(\zeta, X)
$$

for all $X$ near $\tilde{X}$ and $|\zeta|<1$. By (44), $F_{\tilde{p}}(G(0, \tilde{X}))=F_{\tilde{p}}(0)=\tilde{p}$. The regular and general subdifferentials of $\mathfrak{f}$ near $\widetilde{X}$ can be obtained by computing the corresponding objects for the mapping $\mathbf{f} \circ F_{\tilde{p}} \circ G$. The diagram in (6) illustrates the relationship between the mappings $\mathbf{f}, F_{\tilde{p}}, G$, and $\mathfrak{f}$, defined in (2), (17), (43), and (11), respectively. We apply a nonsmooth chain rule to the representation $\mathbf{f} \circ F_{\tilde{p}} \circ G$ to obtain a formula for the subdifferential of $\mathfrak{f}$.

Theorem 11 (Nonsmooth Chain Rule). [19, Theorem 10.6] Suppose $h(x):=$ $\mathrm{g}(H(x))$ for a proper, lsc function $\mathrm{g}: \mathbb{R}^{m} \rightarrow \overline{\mathbb{R}}$ and a smooth mapping $H: \mathbb{R}^{n} \rightarrow$ $\mathbb{R}^{m}$. Then, at any point $\tilde{x} \in \operatorname{dom}(h)=H^{-1}(\operatorname{dom}(\mathrm{g}))$,

$$
\hat{\partial} h(\tilde{x}) \supset H^{\prime}(\tilde{x})^{\star} \hat{\partial} \mathrm{g}(H(\tilde{x})) .
$$

If

$$
\left(y \in \partial^{\infty} \mathrm{g}(H(\tilde{x})) \text { and }\left(H^{\prime}(\tilde{x})\right)^{\star} y=0\right) \Longrightarrow y=0,
$$

then

$$
\partial h(\tilde{x}) \subset\left(H^{\prime}(\tilde{x})\right)^{\star} \partial \mathrm{g}(H(\tilde{x})) \quad \text { and } \quad \partial^{\infty} h(\tilde{x}) \subset\left(H^{\prime}(\tilde{x})\right)^{\star} \partial^{\infty} \mathrm{g}(H(\tilde{x})) .
$$

If, in addition, $\mathrm{g}$ is regular at $H(\tilde{x})$, then $h$ is regular at $\tilde{x}$,

$$
\partial h(\tilde{x})=\left(H^{\prime}(\tilde{x})\right)^{\star} \partial \mathrm{g}(H(\tilde{x})) \quad \text { and } \quad \partial^{\infty} h(\tilde{x})=\left(H^{\prime}(\tilde{x})\right)^{\star} \partial^{\infty} \mathrm{g}(H(\tilde{x})) .
$$

Theorem 12 (Chain Rule for $\mathfrak{f}$ ). Let $f: \mathbb{C} \rightarrow \overline{\mathbb{R}}$ be proper, convex, lsc and let $\mathfrak{f}$ be as in (11). Suppose that $\tilde{X} \in \mathbb{C}^{n \times n}$ is such that the notation of Section 4 holds, $f$ satisfies either (3) or (4) at $\tilde{\lambda}_{j}$ for each $j \in\{1, \ldots, m\}$, and $\Phi_{n}(\tilde{X})$ has active factor $\tilde{p}$ given by (15) and satisfying (29). If $\tilde{\lambda}_{j}$ is nonderogatory for all $j \in\{1, \ldots, m\}$, then

$$
\hat{\partial} \mathfrak{f}(\tilde{X}) \supset\left\{Y \in \mathbb{C}^{n \times n} \mid(0, Y) \in R\left(D_{\tilde{p}}\right)\right\},
$$


where $D_{\tilde{p}}$ and $R$ are as defined in Theorem 3 and (45), respectively. If $f$ satisfies (3) at $\tilde{\lambda}_{j}$ for all $j \in\{1, \ldots, m\}$, then $\mathfrak{f}$ is subdifferentially regular at $\tilde{X}$ with

$$
\begin{aligned}
\partial \mathfrak{f}(\tilde{X}) & =\left\{Y \in \mathbb{C}^{n \times n} \mid(0, Y) \in R\left(D_{\tilde{p}}\right)\right\}, \quad \text { and } \\
\partial^{\infty} \mathfrak{f}(\tilde{X}) & =\left\{Y \in \mathbb{C}^{n \times n} \mid(0, Y) \in R\left(D_{\tilde{p}}^{\infty}\right)\right\},
\end{aligned}
$$

where $D_{\tilde{p}}^{\infty}$ is as defined in Theorem .

Proof. We prove only the second statement since the proof of the first statement is similar. Define $H:\{\zeta:|\zeta|<1\} \times \Omega \rightarrow \mathbb{P}^{\tilde{n}}$ by $H:=F_{\tilde{p}} \circ G$ so that, in particular, $H(0, \tilde{X})=F_{\tilde{p}}(G(0, \tilde{X}))=F_{\tilde{p}}(0)=\tilde{p}$. By Theorem [10, the adjoint of the derivative of $H$ at $(0, \tilde{X})$ is given by

$$
\begin{aligned}
\left(H^{\prime}(\zeta, \tilde{X})\right)^{\star} & =\left(G^{\prime}(\zeta, \tilde{X})\right)^{\star} \circ\left(F_{\tilde{p}}^{\prime}(G(\zeta, \tilde{X}))\right)^{\star} \\
& =R \circ \mathcal{T}_{\tilde{p}} \circ\left(F_{\tilde{p}}^{\prime}(G(\zeta, \tilde{X}))\right)^{\star} \quad \text { by (46). }
\end{aligned}
$$

Therefore,

$$
\begin{array}{rlrl}
\left(H^{\prime}(0, \tilde{X})\right)^{\star} & =R \circ \mathcal{T}_{\tilde{p}} \circ\left(F_{\tilde{p}}^{\prime}(0)\right)^{\star} & \text { by (44) } \\
& =R \circ \mathcal{T}_{\tilde{p}} \circ\left(F_{\tilde{p}}^{\prime}(0)\right)^{-1} \quad \text { by (27). }
\end{array}
$$

Since $\mathcal{T}_{\tilde{p}}, R$ and $F_{\tilde{p}}^{\prime}(0)$ are injective, so is $\left(H^{\prime}(0, \tilde{X})\right)^{\star}$.

Define $\hat{\mathfrak{f}}:\{\zeta:|\zeta|<1\} \times \Omega \rightarrow \overline{\mathbb{R}}$ by

$$
\hat{\mathfrak{f}}(\zeta, X):=(\mathbf{f} \circ H)(\zeta, X) \quad \forall(\zeta, X) \in\{\zeta:|\zeta|<1\} \times \Omega,
$$

so that

$$
\mathfrak{f}(X)=\hat{\mathfrak{f}}(0, X)=\hat{\mathfrak{f}}(\zeta, X)=(\mathbf{f} \circ H)(\zeta, X) \quad \forall(\zeta, X) \in\{\zeta:|\zeta|<1\} \times \Omega .
$$

Take $\mathrm{g}:=\mathbf{f}$ and $h:=\hat{\mathfrak{f}}$ in Theorem 11. Then by Theorems 3 and 11, $\hat{\mathfrak{f}}$ is subdifferentially regular at $(\zeta, \widetilde{X})$ and

$$
\begin{array}{rlrl}
\partial \hat{\mathfrak{f}}(\zeta, \tilde{X}) & =\partial \hat{\mathfrak{f}}(0, \tilde{X}) & & \text { by (49) } \\
& =\left(H^{\prime}(0, \tilde{X})\right)^{\star} \partial \mathbf{f}(H(0, \tilde{X})) & \\
& =R \circ \mathcal{T}_{\tilde{p}} \circ\left(F_{\tilde{p}}^{\prime}(0)\right)^{-1} \partial \mathbf{f}(H(0, \tilde{X})) & & \text { by (48) } \\
& =R \circ \mathcal{T}_{\tilde{p}} \circ\left(F_{\tilde{p}}^{\prime}(0)\right)^{-1} F_{\tilde{p}}^{\prime}(0) \circ \mathcal{T}_{\tilde{p}}^{-1}\left(D_{\tilde{p}}\right) & \text { by (30) } \\
& =R\left(D_{\tilde{p}) .}\right. &
\end{array}
$$

Similarly, $\partial^{\infty} \hat{\mathfrak{f}}(\zeta, \tilde{X})=R\left(D_{\tilde{p}}^{\infty}\right)$. By (49), $\hat{\mathfrak{f}}(\zeta, \widetilde{X})$ is constant on $\{\zeta:|\zeta|<1\}$ so [19. Corollary 10.11] on partial subdifferentiation tells us that the first component of every element of $\partial \hat{\mathfrak{f}}(0, \tilde{X})$ is zero. Hence, the final result also follows from [19], Corollary 10.11].

Remark 13. This result recovers the subdifferential regularity of the spectral abscissa [11, Theorem 8.1] and the formula for its subdifferential in [11, Theorem 7.2] in the case of nonderogatory active eigenvalues.

Corollary 14 (Explicit Subdifferential Representation). Assume the hypotheses of Theorem 12 with (3) holding. Then $Y \in \partial \mathfrak{f}(\tilde{X})$ if and only if there exists $U_{j} \in$ $\mathbb{C}^{n_{j} \times n_{j}}$ for $j \in\{1, \ldots, m\}$ such that

$$
\widetilde{P}^{-*} Y \widetilde{P}=\operatorname{Diag}\left(0_{n_{0} \times n_{0}}, U_{1} \ldots, U_{m}\right),
$$


where $U_{j}$ is lower triangular Toeplitz with diagonal entries $-\mu_{j 1}$, subdiagonal entries $-\mu_{j 2}$, and parameters $\gamma_{j} \geqslant 0$ satisfying $\sum_{j=1}^{m} \gamma_{j}=1$,

$$
\mu_{j 1}=\gamma_{j}\left(\nabla f\left(\tilde{\lambda}_{j}\right) / n_{j}\right) \text { and }\left\langle-\mu_{j 2}, \nabla f\left(\tilde{\lambda}_{j}\right)^{2}\right\rangle_{\mathbb{C}} \leqslant\left(\gamma_{j} / n_{j}\right) f^{\prime \prime}\left(\tilde{\lambda}_{j} ; i \nabla f\left(\tilde{\lambda}_{j}\right), i \nabla f\left(\tilde{\lambda}_{j}\right)\right) .
$$

\section{MATRICES: THE GENERAL CASE}

In Section 5, we use the Arnold form (see Theorem 5) to simultaneously derive a representation for the subdifferential and establish the subdifferential regularity of convexly generated spectral max functions at matrices with nonderogatory active eigenvalues. In this section, we extend techniques developed in [11, Theorem 7.2] for the spectral abscissa to derive a representation for the regular subdifferential of spectral max functions even when some active eigenvalues are derogatory. We do not derive formulas for the general (limiting) subdifferential (see (13)). Formulas in the derogatory case are, for the most part, unknown even for the spectral abscissa1.

6.1. Review of results from [11. Define $\Lambda: \mathbb{C}^{n \times n} \rightarrow \mathbb{C}^{n}$ to be the mapping that takes a matrix $X$ to its vector of eigenvalues, repeated according to algebraic multiplicity and ordered lexicographically (see 3.1). Any function of the form $\psi:=\mathrm{h} \circ \Lambda$, where $\mathrm{h}: \mathbb{C}^{n} \rightarrow \overline{\mathbb{R}}$ is invariant under permutations of its arguments, is a spectral function (see \$1).

Now assume that the set $\widetilde{\Xi}=\left\{\tilde{\lambda}_{1}, \ldots, \tilde{\lambda}_{m}\right\}$ in (33) is the complete set of distinct eigenvalues of $\tilde{X} \in \mathbb{C}^{n \times n}$ with algebraic multiplicities $n_{1}, \ldots, n_{m}$ (so that $n=$ $\sum_{j=1}^{m} n_{j}$ ) and geometric multiplicities $q_{1}, \ldots, q_{m}$. We use the Jordan form notation described in (34) and (35), where the matrix $\widetilde{B}$ is no longer present. Recall the following results from 11 .

Theorem 15. [11, Theorems 4.1 and 4.2] Let $\tilde{X}$ be as given above and let $\psi$ be a spectral function. If $Y$ is a subgradient or horizon subgradient of $\psi$ at $\tilde{X}$, then

$$
W:=\widetilde{P}^{-*} Y \widetilde{P}^{*}
$$

satisfies

$$
W=\operatorname{Diag}\left(W_{1}, \ldots, W_{m}\right)
$$

where

$$
W_{j}:=\left[\begin{array}{ccc}
W_{j}^{(11)} & \cdots & W_{j}^{\left(1 q_{j}\right)} \\
\vdots & \ddots & \vdots \\
W_{j}^{\left(q_{j} 1\right)} & \cdots & W_{j}^{\left(q_{j} q_{j}\right)}
\end{array}\right],
$$

with $W_{j}^{(r s)}$ a rectangular $m_{j r} \times m_{j s}$ lower triangular Toeplitz matrix for $r=$ $1, \ldots, q_{j}, s=1, \ldots, q_{j}, j=1, \ldots, m$.

If $Y$ is further assumed to be a regular subgradient of $\psi$ at $\tilde{X}$, then

$$
W_{j}=\operatorname{Diag}\left(W_{j}^{(11)}, \ldots, W_{j}^{\left(q_{j} q_{j}\right)}\right),
$$

where $W_{j}^{(k k)}$ is an $m_{j k} \times m_{j k}$ lower triangluar Toeplitz matrix with diagonal $\theta_{j 1}$ and subdiagonals $\theta_{j s}, s=2, \ldots, m_{j k}, k=1, \ldots, q_{j}, j=1, \ldots, m$, and $\sum_{k=1}^{q_{j}} m_{j k}=n_{j}$.

\footnotetext{
${ }^{1}$ Grundel and Overton derive the general subdifferential in the simplest derogatory, defective case when $n=3$ for the spectral abscissa in [14]. The nondefective (or semisimple) case is treated in [11 Theorem 8.3].
} 
Remark 16. By lower triangular Toeplitz, we mean that the value of the $k, \ell$ entry of $W_{j}^{(r s)}$ depends only on the difference $k-\ell$ (is constant along the diagonals), and is zero if $k<\ell$ or $m_{j r}-k>m_{j s}-\ell$ (is zero above the main diagonal, drawn from either the top left or bottom right of the block).

Observe that $\Phi_{n}$ (see (5) ) is smooth since each coefficient is a polynomial in the entries of $X$. The next result provides an expression for one-sided $\mathbb{C}$-derivatives of $\Phi_{n}$ with respect to a given direction.

Lemma 17. 11, Lemma 7.1] Let $\tilde{X} \in \mathbb{C}^{n \times n}$ and $Z \in \mathbb{C}^{n \times n}$ be given, and assume $\tilde{X}$ has Jordan form (34). Define $p \in \mathbb{M}^{n}$ by

$$
p:=\Phi_{n}(\tilde{X})=\prod_{j=1}^{m} e_{\left(n_{j}, \tilde{\lambda}_{j}\right)},
$$

where $\Phi_{n}$ and $e_{\left(\ell, \lambda_{0}\right)}$ are defined in (5) and (16), respectively. Set

$$
V:=\widetilde{P} Z \widetilde{P}^{-1},
$$

and let $V_{j j}$ be the $n_{j} \times n_{j}$ diagonal block of $V$ corresponding to block $J_{j}$ of the matrix $J$ defined in (34). Then the action of the $\mathbb{C}$-derivative $\Phi_{n}^{\prime}(\tilde{X})$ is

$$
\Phi_{n}^{\prime}(\tilde{X}) Z=-\sum_{j=1}^{m}\left(\prod_{k=1, k \neq j}^{m} e_{\left(n_{k}, \tilde{\lambda}_{k}\right)}\right)\left(\sum_{\ell=1}^{m_{j}} \operatorname{tr}\left(N_{[j]}^{\ell-1} V_{j j}\right) e_{\left(n_{j}-\ell, \tilde{\lambda}_{j}\right)}\right)
$$

for all $Z \in \mathbb{C}^{n \times n}$, where

$$
N_{[j]}:=\operatorname{Diag}\left(N_{j 1}, \ldots, N_{j q_{j}}\right)
$$

with $N_{j k}$ defined in (35). $N_{[j]}:=\operatorname{Diag}\left(N_{j 1}, \ldots, N_{j q_{j}}\right)$ with $N_{j k}$ defined in (35).

Theorem 18. Suppose $\mathfrak{f}$ is as in (1), where

$$
\nabla f \text { is continuous at } \tilde{\lambda}_{j} \text { with } \nabla f\left(\tilde{\lambda}_{j}\right) \neq 0 \forall j \in \mathcal{I}_{\mathfrak{f}}(\tilde{X}) \text {. }
$$

Define

$$
\sigma_{j}:= \begin{cases}\theta_{j 1} / \nabla f\left(\tilde{\lambda}_{j}\right), & j \in \mathcal{I}_{\mathfrak{f}}(\tilde{X}) \\ 0, & j \notin \mathcal{I}_{\mathfrak{f}}(\tilde{X}),\end{cases}
$$

where $\theta_{j 1}$ is given in (53), and set $\sigma:=\left[\sigma_{1}, \ldots, \sigma_{1}, \ldots, \sigma_{m}, \ldots, \sigma_{m}\right]^{T} \in \mathbb{C}^{n}$, where each $\sigma_{j}$ is repeated $n_{j}$ times. Let $\tilde{X}$ have Jordan form (34), set

$$
\triangle^{n-1}:=\left\{\gamma \in \mathbb{R}^{n} \mid 0 \leqslant \gamma_{i}, i=1, \ldots, n, \sum_{i=1}^{n} \gamma_{i}=1\right\},
$$

and assume that $Y$ is a regular subgradient for $\mathfrak{f}$ at $\tilde{X}$. Then $Y$ satisfies (52) and (53), and we have the following:

(a) [11, Theorem 6.1] For all $j \in\{1, \ldots, m\}$,

$$
j \notin \mathcal{I}_{\mathfrak{f}}(\tilde{X}) \quad \Longrightarrow \quad W_{j}=0,
$$

where $W_{j}$ is given in (53).

(b) [11, Theorem 5.2, Equations 6.5, 6.6] We have $\sigma \in \triangle^{n-1}$ with $\sigma_{j}=0$ if $j \notin$ $\mathcal{I}_{\mathfrak{f}}(\tilde{X})$. In particular, this implies that

$$
\sigma_{j} \geqslant 0 \quad \text { and } \quad \sum_{j \in \mathcal{I}_{\mathfrak{f}}(\widetilde{X})} n_{j} \sigma_{j}=1 .
$$


(c) [11, Theorem 5.3] If, in addition, the second $\mathbb{R}$-derivative of $f$ is continuous at each $\tilde{\lambda}_{j}$ for $j \in \mathcal{I}_{\mathfrak{f}}(\tilde{X})$, then

$$
\left\langle\theta_{j 2},\left(\nabla f\left(\tilde{\lambda}_{j}\right)\right)^{2}\right\rangle_{\mathbb{C}} \geqslant-\sigma_{j} \eta_{j} \quad \text { whenever } m_{j} \geqslant 2,
$$

where $\sigma_{j}$ is given in (58), $\theta_{j 2}$ is given in (53), $m_{j}$ is given in (36), and

$$
\eta_{j}:=f^{\prime \prime}\left(\tilde{\lambda}_{j} ; i \nabla f\left(\tilde{\lambda}_{j}\right), i \nabla f\left(\tilde{\lambda}_{j}\right)\right) .
$$

6.2. The regular subdifferential and its recession cone. The main result of Section 6 now follows.

Theorem 19 (Regular subdifferential formula). Let $\tilde{X}$ have Jordan form (34) with the distinct eigenvalues $\widetilde{\Xi}$ having geometric multiplicities $q_{1}, \ldots, q_{m}$. Suppose $f$ satisfies (3) at each $\tilde{\lambda}_{j}$ for $j \in \mathcal{I}_{\mathfrak{f}}(\tilde{X})$ and (57) holds. Then $Y \in \hat{\partial} \mathfrak{f}(\tilde{X})$ if and only if $Y$ satisfies (50)-(53) and (60)-(62) .

Proof. We follow the pattern of proof established in [11, Theorem 7.2] for the spectral abscissa. Let $Y \in \hat{\partial} \mathfrak{f}(\tilde{X})$. By Theorems [15 and 18, $Y$ satisfies (50)-(153) and (60)- -621 , respectively.

Next suppose that $Y \in \mathbb{C}^{n \times n}$ satisfies conditions (50)-(53) and (60)-(62). We prove that $Y$ is a regular subgradient, that is,

$$
\langle Y, Z\rangle_{\mathbb{C}^{n \times n}} \leqslant \mathrm{~d} \mathfrak{f}(\tilde{X})(Z), \quad \text { for all } \quad Z \in \mathbb{C}^{n \times n} .
$$

By applying the chain rule (see Theorem[11), we obtain

$$
\mathrm{d} \mathfrak{f}(\tilde{X})(Z)=\mathrm{d}\left(\mathbf{f} \circ \Phi_{n}\right)(\tilde{X})(Z) \geqslant \mathrm{d} \mathbf{f}\left(\Phi_{n}(\tilde{X})\right)\left(\Phi_{n}^{\prime}(\tilde{X}) Z\right),
$$

where the action $\Phi_{n}^{\prime}(\tilde{X}) Z$ is given in (55) and $\mathbf{f}$ is defined in (22). Set $p:=\Phi_{n}(\tilde{X})$ in (65) to obtain

By (50)- $-(53)$ and (60),

$$
\mathrm{d} \mathfrak{f}(\tilde{X})(Z) \geqslant \mathrm{d} \mathbf{f}(p)\left(\Phi_{n}^{\prime}(\tilde{X}) Z\right) .
$$

$$
\langle Y, Z\rangle_{\mathbb{C}^{n \times n}}=\langle W, V\rangle_{\mathbb{C}^{n \times n}}=\sum_{j \in \mathcal{I}_{\mathfrak{f}}(\widetilde{X})} \sum_{\ell=1}^{m_{j}} \operatorname{Re}\left(\overline{\theta_{j \ell}} \operatorname{tr}\left(N_{[j]}^{\ell-1} V_{j j}\right)\right),
$$

where $V$ is defined in (154). Set $\omega_{j \ell}:=-\operatorname{tr}\left(N_{[j]}^{\ell-1} V_{j j}\right)$ for $\ell=1, \ldots, m_{j}, j \in \mathcal{I}_{\mathfrak{f}}(\tilde{X})$. Then

$$
\langle W, V\rangle_{\mathbb{C}^{n \times n}}=\sum_{j \in \mathcal{I}_{\mathfrak{f}}(\widetilde{X})} \sum_{\ell=1}^{m_{j}} \operatorname{Re}\left(\overline{\theta_{j \ell}}\left(-\omega_{j \ell}\right)\right) .
$$

If either (31) or (32) in Theorem 4 fails for some $j \in \mathcal{I}_{\mathfrak{f}}(\tilde{X})$, then the subderivative $\mathrm{d} \mathbf{f}(p)\left(\Phi_{n}^{\prime}(\tilde{X}) Z\right)$ is infinite, in which case (64) is trivially satisfied. If (31) and (32) in Theorem 4 hold for all $j \in \mathcal{I}_{\mathfrak{f}}(\tilde{X})$, then

$$
\begin{aligned}
\langle Y, Z\rangle_{\mathbb{C}^{n \times n}} & =\sum_{j \in \mathcal{I}_{\mathfrak{f}}(\widetilde{X})}\left(\operatorname{Re}\left(\overline{\theta_{j 1}}\left(-\omega_{j 1}\right)\right)+\operatorname{Re}\left(\overline{\theta_{j 2}}\left(-\omega_{j 2}\right)\right)\right), \\
& =\sum_{j \in \mathcal{I}_{\mathfrak{f}}(\widetilde{X})} \operatorname{Re}\left(\overline{\sigma_{j} \nabla f\left(\tilde{\lambda}_{j}\right)}\left(-\omega_{j 1}\right)\right)-\operatorname{Re}\left(\overline{\theta_{j 2}} \omega_{j 2}\right) \text { by } \overline{(58)}, \\
& =\sum_{j \in \mathcal{I}_{\mathfrak{f}}(\widetilde{X})} \sigma_{j} \operatorname{Re}\left(\overline{\nabla f\left(\tilde{\lambda}_{j}\right)}\left(-\omega_{j 1}\right)\right)-\operatorname{Re}\left(\overline{\theta_{j 2}} \omega_{j 2}\right) \text { by ([61), } \\
& =\sum_{j \in \mathcal{I}_{\mathfrak{f}}(\widetilde{X})} \sigma_{j} f^{\prime}\left(\tilde{\lambda}_{j} ;-\omega_{j 1}\right)-\operatorname{Re}\left(\overline{\theta_{j 2}} \omega_{j 2}\right) \quad \text { by (14), }
\end{aligned}
$$

where the second term in each line does not appear if $m_{j}=1$ (see (36i)). Recall that for all $j \in \mathcal{I}_{\mathfrak{f}}(\tilde{X}), \omega_{j 2}$ satisfies (31), which, since $f$ satisfies (3) at $\tilde{\lambda}_{j}$, is equivalent to

$$
\omega_{j 2}=t_{j}\left(\nabla f\left(\tilde{\lambda}_{j}\right)\right)^{2} \quad \text { for some } t_{j} \geqslant 0
$$


by [9. Lemma 4]. Therefore,

$$
\begin{array}{rlrl}
\left\langle\theta_{j 2}, f^{\prime}\left(\tilde{\lambda}_{j}\right)^{2}\right\rangle_{\mathbb{C}} & \geqslant-\sigma_{j} \eta_{j} & & \text { by (62), which implies } \\
\operatorname{Re}\left(\overline{\theta_{j 2}} \omega_{j 2}\right) & \geqslant-t_{j} \sigma_{j} \eta_{j} & & \text { by ([68), which implies } \\
-\operatorname{Re}\left(\overline{\theta_{j 2}} \omega_{j 2}\right) & \leqslant t_{j} \sigma_{j} f^{\prime \prime}\left(\tilde{\lambda}_{j} ; \mathrm{i} f^{\prime}\left(\tilde{\lambda}_{j}\right), \mathrm{i} f^{\prime}\left(\tilde{\lambda}_{j}\right)\right), & & \text { by ([63), which implies } \\
-\operatorname{Re}\left(\overline{\theta_{j 2}} \omega_{j 2}\right) & \leqslant \sigma_{j} f^{\prime \prime}\left(\tilde{\lambda}_{j} ; \sqrt{-\omega_{j 2}}, \sqrt{-\omega_{j 2}}\right) . &
\end{array}
$$

Plugging (69) into (67) yields

$$
\begin{aligned}
\langle Y, Z\rangle_{\mathbb{C}^{n \times n}} & =\sum_{j \in \mathcal{I}_{\mathfrak{f}}(\widetilde{X})}\left(\sigma_{j} f^{\prime}\left(\tilde{\lambda}_{j} ;-\omega_{j 1}\right)-\operatorname{Re}\left(\overline{\theta_{j 2}} \omega_{j 2}\right)\right) \\
& \leqslant \sum_{j \in \mathcal{I}_{\mathfrak{f}}(\widetilde{X})}\left(\sigma_{j} f^{\prime}\left(\tilde{\lambda}_{j} ;-\omega_{j 1}\right)+\sigma_{j} f^{\prime \prime}\left(\tilde{\lambda}_{j} ; \sqrt{-\omega_{j 2}}, \sqrt{-\omega_{j 2}}\right)\right) \\
& =\sum_{j \in \mathcal{I}_{\mathfrak{f}}(\widetilde{X})} \sigma_{j} n_{j}\left(f^{\prime}\left(\tilde{\lambda}_{j} ;-\omega_{j 1}\right)+f^{\prime \prime}\left(\tilde{\lambda}_{j} ; \sqrt{-\omega_{j 2}}, \sqrt{-\omega_{j 2}}\right)\right) / n_{j} \\
& \leqslant \max _{j \in \mathcal{I}_{\mathfrak{f}}(\widetilde{X})}\left\{\left(f^{\prime}\left(\tilde{\lambda}_{j} ;-\omega_{j 1}\right)+f^{\prime \prime}\left(\tilde{\lambda}_{j} ; \sqrt{-\omega_{j 2}}, \sqrt{-\omega_{j 2}}\right)\right) / n_{j}\right\} \text { by (61) } \\
& =\operatorname{df}(p)\left(\Phi_{n}^{\prime}(\tilde{X}) Z\right),
\end{aligned}
$$

where the last equality holds by Theorem 4. Combining this with (66) gives (664).

Remark 20. Theorem 19 establishes the formula for the regular subdifferential of $\mathfrak{f}$ at a matrix with any number of derogatory or nonderogatory active eigenvalues. The theorem recovers the formula for the regular subdifferential in the case where all active eigenvalues are nonderogatory (see Corollary 14). In Section 7, it is shown that although Theorem 19 does not directly apply to the spectral radius, it can still be used to derive its regular subdifferential.

Theorem 21 (Recession cone of the regular subdifferential). Assume the hypotheses of Theorem 19. Then $Y \in \hat{\partial} \mathfrak{f}(\tilde{X})^{\infty}$ if and only if $Y$ satisfies (50)-(53), (60), and, for $j \in \mathcal{I}_{\mathfrak{f}}(\tilde{X})$, the diagonal entries $\theta_{j 1}$ in (53) satisfy

$$
\theta_{j 1}=0,
$$

and, if in addition $m_{j} \geqslant 2$ (see (36)), the subdiagonal entries $\theta_{j 2}$ in (53) satisfy

$$
\left\langle\theta_{j 2},\left(\nabla f\left(\tilde{\lambda}_{j}\right)\right)^{2}\right\rangle_{\mathbb{C}} \geqslant 0 .
$$

Proof. First suppose $Y \in \hat{\partial} \mathfrak{f}(\tilde{X})^{\infty}$. Since $\hat{\partial} \mathfrak{f}(\tilde{X})$ is convex, $Z+t Y \in \hat{\partial} \mathfrak{f}(\tilde{X})$ for all $Z \in \hat{\partial} \mathfrak{f}(\tilde{X})$ and $t \geqslant 0$ by the definition of recession cone. By Theorem [15, both $\widetilde{P}^{-*} Z \widetilde{P}^{*}$ and $\widetilde{P}^{-*}(Z+t Y) \widetilde{P}^{*}$ satisfy (50)-(53) for all $Z \in \hat{\partial} \mathfrak{f}(\tilde{X})$ and $t \geqslant 0$. In particular, both $\widetilde{P}^{-*} Z \widetilde{P}^{*}$ and $\widetilde{P}^{-*}(Z+t Y) \widetilde{P}^{*}$ are block diagonal matrices, with each block a lower triangular Toeplitz matrix where the blocks corresponding to the same eigenvalue have the same diagonal entries. Therefore, $\widetilde{P}^{-*} Y \widetilde{P}^{*}$ has the block structure specified in (50)-(53). By Theorem [18, $Y$ also satisfies (60).

For $Z \in \hat{\partial} \mathfrak{f}(\tilde{X})$, denote the diagonal entries of the $j$ th block of $\widetilde{P}^{-*} Z \widetilde{P}^{*}$ by $z_{j 1}$. By Theorem [18, $Z+t Y$ satisfies (61) for all $t \geqslant 0$, that is,

$$
\sum_{j \in \mathcal{I}_{\mathfrak{f}}(\widetilde{X})} n_{j}\left(z_{j 1}+t \theta_{j 1}\right) / \nabla f\left(\tilde{\lambda}_{j}\right)=1
$$

and $\left(z_{j 1}+t \theta_{j 1}\right) / \nabla f\left(\tilde{\lambda}_{j}\right) \geqslant 0$ for all $j \in \mathcal{I}_{\mathfrak{f}}(\tilde{X})$ and $t \geqslant 0$. Therefore, $\theta_{j 1}=0$ for all $j \in \mathcal{I}_{\mathfrak{f}}(\tilde{X})$, proving (20). If, in addition, $m_{j} \geqslant 2$ (see (36) ), denote the subdiagonal 
entries of $\widetilde{P}^{-*} Z \widetilde{P}^{*}$ by $z_{j 2}$. By Theorem $18, t Y$ satisfies (62), that is,

$$
\left\langle\left(z_{j 2}+t \theta_{j 2}\right),\left(\nabla f\left(\tilde{\lambda}_{j}\right)\right)^{2}\right\rangle_{\mathbb{C}} \geqslant-\left(z_{j 1}+t \theta_{j 1}\right) \eta_{j} / \nabla f\left(\tilde{\lambda}_{j}\right)
$$

for all $j \in \mathcal{I}_{\mathfrak{f}}(\tilde{X})$ and $t \geqslant 0$. Since $\theta_{j 1}=0$, this becomes

$$
\left\langle z_{j 2},\left(\nabla f\left(\tilde{\lambda}_{j}\right)\right)^{2}\right\rangle_{\mathbb{C}}+t\left\langle\theta_{j 2},\left(\nabla f\left(\tilde{\lambda}_{j}\right)\right)^{2}\right\rangle_{\mathbb{C}} \geqslant-z_{j 1} \eta_{j} / \nabla f\left(\tilde{\lambda}_{j}\right)
$$

for all $t \geqslant 0$ and $Z \in \hat{\partial} \mathfrak{f}(\tilde{X})$. Since, by (62),

$$
\left\langle z_{j 2},\left(\nabla f\left(\tilde{\lambda}_{j}\right)\right)^{2}\right\rangle_{\mathbb{C}} \geqslant-z_{j 1} \eta_{j} / \nabla f\left(\tilde{\lambda}_{j}\right)
$$

for all $j \in \mathcal{I}_{\mathfrak{f}}(\tilde{X})$ with $m_{j} \geqslant 2$, (71) follows.

Now suppose $Y \in \mathbb{C}^{n \times n}$ satisfies (50)-(53), (60), (700), and, for $j \in \mathcal{I}_{\mathfrak{f}}(\tilde{X})$ with $m_{j} \geqslant 2$, (71). Then for any $t \geqslant 0$ and $Z \in \hat{\partial} \mathfrak{f}(\tilde{X})$, the matrix $Z+t Y$ satisfies the conditions of Theorem [19, which implies that $Z+t Y \in \hat{\partial} \mathfrak{f}(\tilde{X})$, proving the reverse inclusion.

6.3. Nonderogatory active eigenvalues are necessary for regularity. Theorem 12 shows that nonderogatory active eigenvalues are sufficient for the subdifferential regularity of $\mathfrak{f}$. We now show this condition is also necessary.

Lemma 22. In (33), let $\widetilde{\Xi}$ be the complete set of distinct eigenvalues of $\tilde{X} \in \mathbb{C}^{n \times n}$, and suppose $f$ satisfies (57). If $\tilde{\lambda}_{j}$ is derogatory for some $j \in \mathcal{I}_{\mathfrak{f}}(\tilde{X})$, then $\partial \mathfrak{f}(\tilde{X}) \supsetneq$ $\hat{\partial} \mathfrak{f}(\tilde{X})$.

Proof. We follow the method of proof in [11, Theorem 8.2] for the spectral abscissa. Let $j \in \mathcal{I}_{\mathfrak{f}}(\tilde{X})$ be such that $\tilde{\lambda}_{j}$ is derogatory. Then the geometric multiplicity of $\tilde{\lambda}_{j}$ is at least 2 . Since $f$ is differentiable at $\tilde{\lambda}_{j}, \tilde{\lambda}_{j} \in \operatorname{int}(\operatorname{dom}(f))$. This together with the fact that $\nabla f\left(\tilde{\lambda}_{j}\right) \neq 0$ implies $\tilde{\lambda}_{j}$ is not a local maximizer of $f$. Therefore, there exists $\lambda^{\nu} \rightarrow \tilde{\lambda}_{j}$ with $f\left(\lambda^{\nu}\right)>f\left(\tilde{\lambda}_{j}\right)$. Set $\beta^{\nu}:=\lambda^{\nu}-\tilde{\lambda}_{j}$ and define $X^{\nu}:=\widetilde{P}^{-1}\left(J+\beta^{\nu} E\right) \widetilde{P}$ where $\widetilde{P}$ and $J$ are defined in (34), and the entries of $E$ are one in the $m_{j 1}$ diagonal positions corresponding to the Jordan sub-block $J_{j}^{(1)}$ with all other entries zero. Clearly $X^{\nu} \rightarrow \tilde{X}$. The only active eigenvalue of $X^{\nu}$ is $\lambda^{\nu}$, with multiplicity $m_{j 1}$, and $J+\beta^{\nu} E$ is the Jordan form of $X^{\nu}$, up to re-ordering of the Jordan blocks. By Theorem 19, $\hat{\partial} \mathfrak{f}\left(X^{\nu}\right)$ includes the matrix $\left(\nabla f\left(\lambda^{\nu}\right) / m_{j 1}\right) \widetilde{P}^{*} E \widetilde{P}^{-*}$ for all $\nu$, which implies $\left(\nabla f\left(\tilde{\lambda}_{j}\right) / m_{j 1}\right) \widetilde{P}^{*} E \widetilde{P}^{-*} \in \partial \mathfrak{f}(\tilde{X})$. However, $\left(\nabla f\left(\tilde{\lambda}_{j}\right) / m_{j 1}\right) \widetilde{P}^{*} E \widetilde{P}^{-*} \notin \hat{\partial} \mathfrak{f}(\tilde{X})$ since $\left(\nabla f\left(\tilde{\lambda}_{j}\right) / m_{j 1}\right) \widetilde{P}^{*} E \widetilde{P}^{-*}$ does not satisfy the conditions in Theorem 19 because the diagonal entries of $W_{j}$ are 0 for blocks $m_{j 2}$ to $m_{j q_{j}}$ but are nonzero for block $m_{j 1}$. Therefore, $\partial \mathfrak{f}(\tilde{X}) \supsetneq \hat{\partial} \mathfrak{f}(\tilde{X})$.

The following result follows as an immediate consequence of Theorem 12 and the preceding lemma.

Theorem 23. Let $f: \mathbb{C} \rightarrow \overline{\mathbb{R}}$ be proper, convex, lsc, and let $\mathfrak{f}$ be the associated spectral max function as in (11). Suppose that $\widetilde{X} \in \mathbb{C}^{n \times n} f$ satisfies (3) at all active eigenvalues of $\tilde{X}$. Then $\mathfrak{f}$ is subdifferentially regular at $\widetilde{X}$ if and only if the active eigenvalues of $\tilde{X}$ are nonderogatory. 


\section{Application to the spectral Radius}

Let $r: \mathbb{C} \rightarrow \mathbb{R}$ be the complex modulus map $r(\zeta):=|\zeta|$. The polynomial radius $\mathbf{r}: \mathbb{P}^{n} \rightarrow \overline{\mathbb{R}}$ and the spectral radius $\rho: \mathbb{C}^{n \times n} \rightarrow \overline{\mathbb{R}}$ are given by $\mathbf{r}(p)=$ $\max \{r(\lambda) \mid p(\lambda)=0\}$ and $\rho(X)=\max \{r(\lambda) \mid \operatorname{det}(\lambda I-X)=0\}$, respectively. The results of the previous sections do not directly apply to $\rho$ as (3) is not satisfied, since $r^{\prime \prime}(\lambda ; t \lambda, t \lambda)=0$ for all $\lambda \neq 0$ and $t \in \mathbb{R}$, and $r^{\prime}(0)$ does not exist (see 92.2 ). As in [9], we overcome these hurdles by introducing the function $r_{2}(\zeta):=|\zeta|^{2} / 2$. Since $\nabla^{2} r_{2}(\lambda)$ is the identity map on $\mathbb{C}, r_{2}$ satisfies (3) on all of $\mathbb{C}$. Set $\rho_{2}(\widetilde{X}):=$ $\max \left\{|\lambda|^{2} / 2 \mid \operatorname{det}(\lambda I-\tilde{X})=0\right\}$. The strategy is to first consider the case $\rho(\tilde{X})>0$ using $\rho_{2}$ and then to directly consider the case $\rho(\tilde{X})=0$. When $\rho(\tilde{X})>0$, the relationship between the regular subdifferentials of $\rho$ and $\rho_{2}$ is a consequence of the following result.

Lemma 24. Let $\tilde{X} \in \mathbb{C}^{n \times n}$ be such that $\rho(\tilde{X})>0$. Then

$$
\begin{aligned}
& T_{\operatorname{epi}(\rho)}(\tilde{X}, \mu)=\left\{(V, \eta / \mu) \mid(V, \eta) \in T_{\operatorname{epi}\left(\rho_{2}\right)}\left(\tilde{X}, \mu^{2} / 2\right)\right\}, \text { and } \\
& \widehat{N}_{\operatorname{epi}(\rho)}(\tilde{X}, \mu)=\left\{(W, \mu \tau) \mid(W, \tau) \in \widehat{N}_{\operatorname{epi}\left(\rho_{2}\right)}\left(\tilde{X}, \mu^{2} / 2\right)\right\} .
\end{aligned}
$$

Proof. Recall the definitions of the tangent and regular normal cones given in (11) and (12), respectively. The elementary proof of the tangent cone equality is identical to that in [9, Lemma 7] with polynomials replaced by matrices and the root max function replaced by the spectral max function. The regular normal cone expression follows by taking polars (12).

Theorem $25(\rho(\tilde{X})>0)$. Let $\tilde{X}$ have Jordan form (34) with $\rho(\tilde{X})>0$. Then $Y \in \hat{\partial} \rho(\tilde{X})$ if and only if $Y$ satisfies (50) -(53), (160), and, for $j \in \mathcal{I}_{\rho}(\tilde{X})$, the diagonal entries $\theta_{j 1}$ of $W_{j}$ satisfy

$$
\theta_{j 1} / \tilde{\lambda}_{j} \in \mathbb{R}, \quad \theta_{j 1} / \tilde{\lambda}_{j} \geqslant 0, \text { and } \sum_{j \in \mathcal{I}_{\rho}(\widetilde{X})} n_{j} \theta_{j 1}\left|\tilde{\lambda}_{j}\right| / \tilde{\lambda}_{j}=1,
$$

and, if in addition $m_{j} \geqslant 2$ (see (36)), the subdiagonal entries $\theta_{j 2}$ of $W_{j}$ satisfy

$$
\operatorname{Re}\left(\overline{\theta_{j 2}} \tilde{\lambda}_{j}^{2}\right) \geqslant-\theta_{j 1}\left|\tilde{\lambda}_{j}\right|^{2} / \tilde{\lambda}_{j}
$$

Moreover, $Y \in \hat{\partial} \rho(\tilde{X})^{\infty}$ if and only if $Y$ satisfies (501)-(53), $\theta_{j 1}=0$ for all $j \in$ $\{1, \ldots m\}$, and, for $j \in \mathcal{I}_{\rho}(\widetilde{X})$ with $m_{j} \geqslant 2, \operatorname{Re}\left(\bar{\theta}_{j 2} \tilde{\lambda}_{j}^{2}\right) \geqslant 0$. Finally, $\rho$ is subdifferentially regular at $\tilde{X}$ if all active eigenvalues are nonderogatory.

Remark 26. The proof uses the following formula for the subderivative of the polynomial radius when $p \in \mathbb{M}^{n}$ and $\mathbf{r}(p)>0$ :

$$
\mathrm{d} \mathbf{r}(\tilde{p})(v)=\max _{j \in \mathcal{I}_{\mathbf{r}}(\tilde{p})}\left\{\left(\left|\omega_{j 2}\right|-\operatorname{Re}\left(\tilde{\tilde{\lambda}}_{j} \omega_{j 1}\right)\right) /\left(\left|\tilde{\lambda}_{j}\right| n_{j}\right)\right\},
$$

where $v=\sum_{j=1}^{m} \prod_{k \neq j} e_{\left(n_{k}, \tilde{\lambda}_{k}\right)} \sum_{s=1}^{n_{j}} \omega_{j s} e_{\left(n_{j}-s, \tilde{\lambda}_{j}\right)}$, provided that $\omega_{j 2} \in \operatorname{cone}\left(\tilde{\lambda}_{j}^{2}\right)$ and $\omega_{j s}=0$ for $s=3, \ldots, n_{j}$; otherwise, $d \mathbf{r}(\tilde{p})(v)=+\infty$.

Proof. We first address the regular subdifferential formula. We omit the full proof since it parallels the proof of Theorem[19. We describe a few key ingredients specific to the radius. First, the following formulas are needed (see [9, Section 5])

$$
\nabla r(\zeta)=\zeta /|\zeta| \quad \forall \zeta \in \mathbb{C} \backslash\{0\},
$$


and

$$
r^{\prime \prime}(\zeta ; \delta, \delta)=(1 /|\delta|)\left(|\delta|^{2}-\langle\zeta /|\zeta|, \delta\rangle_{\mathbb{C}}^{2}\right) \quad \forall \zeta, \delta \in \mathbb{C} \backslash\{0\} .
$$

Applying these formulas to the quantity $\eta_{j}$ in (63) imply that $\eta_{j}=1 /\left|\tilde{\lambda}_{j}\right|$, that display (61) is equivalent to (72), and that display (62) is equivalent to (73). Second, note that the hypotheses of Theorems 15] and 18, which are applied in the proof of Theorem 19, do not require that $r$ satisfy (3). They only require that $r$ be differentiable, which is the case on $\mathbb{C} \backslash\{0\}$. Finally, the explicit formula for the subderivative of $\mathbf{r}$ in Remark 26 is utilized.

Next, the formula for the recession cone of the regular subdifferential parallels that of Theorem 21 .

Lastly, we discuss the subdifferential regularity in the case where $\tilde{X}$ has nonderogatory active eigenvalues and $\rho(\tilde{X})>0$. The map $r_{2}: \mathbb{C} \rightarrow \mathbb{R}$ satisfies (3) at $\lambda$ for all $\lambda \in \mathbb{C}$. Therefore $\rho_{2}$ is subdifferentially regular at $\tilde{X}$ by Theorem 12 Using Lemma 24, it is straightforward to establish the subdifferential regularity of $\rho$ (see 19. Definitions 6.4 and 7.25] - Clarke regularity of the epigraph is subdifferential regularity).

When $\rho(\tilde{X})=0, \tilde{X}$ has a single eigenvalue, 0 , with algebraic multiplicity $n$. For this reason, we suppress the index " $j$ " in (50)-(53).

Theorem $27(\rho(\tilde{X})=0)$. Let $\tilde{X} \in \mathbb{C}^{n \times n}$ be such that $\rho(\tilde{X})=0$. Then $Y \in \hat{\partial} \rho(\tilde{X})$ if and only if $Y$ satisfies (501) -(53) and

$$
\left|\theta_{1}\right| \leqslant 1 / n
$$

where $\theta_{1}$ is the diagonal entry of $P^{-*} Y \widetilde{P}^{*}$. Moreover, $Y \in \hat{\partial} \rho(\tilde{X})^{\infty}$ if and only if $Y$ satisfies (50)-(53) and $\theta_{1}=0$. Finally, $\rho$ is subdifferentially regular at $\tilde{X}$ if $\tilde{X}$ is nonderogatory.

Proof. We first prove the regular subdifferential formula. Note that the characteristic polynomial of $\tilde{X}$ is $e_{(n, 0)}$. We will use the fact that

$$
Y \in \hat{\partial} \rho(\tilde{X}) \quad \text { if and only if } \quad\langle Y, Z\rangle \leqslant \mathrm{d} \rho(\tilde{X})(Z) \text { for all } Z \in \mathbb{C}^{n \times n} .
$$

Suppose $Y \in \mathbb{C}^{n \times n}$ satisfies (50)-(53) and (75). By the chain rule [19, Theorem 10.6],

$$
\mathrm{d} \rho(\tilde{X})(Z) \geqslant \mathrm{d} \mathbf{r}\left(\Phi_{n}(\tilde{X})\right)\left(\Phi_{n}^{\prime}(\tilde{X}) Z\right)=\mathrm{d} \mathbf{r}\left(e_{(n, 0)}\right)\left(\Phi_{n}^{\prime}(\tilde{X}) Z\right)
$$

for all $Z \in \mathbb{C}^{n \times n}$. By [9, Theorem 11],

$$
\operatorname{dr}\left(e_{(n, 0)}\right)\left(\Phi_{n}^{\prime}(\tilde{X}) Z\right)= \begin{cases}|\operatorname{tr}(V)| / n & \text { if } \operatorname{tr}\left(N^{s-1} V\right)=0 \text { for } s=2, \ldots, n \\ +\infty & \text { otherwise }\end{cases}
$$

where $V$ is given in (54) and $N \in \mathbb{C}^{n \times n}$ is the nilpotent matrix in (56) (with $j$ 's suppressed). The inequality in (76) is trivially satisfied if $\operatorname{tr}\left(N^{s-1} V\right) \neq 0$ for some $s=2, \ldots, n$ since in this case $d \mathbf{r}\left(e_{(n, 0)}\right)\left(\Phi_{n}^{\prime}(\tilde{X}) Z\right)$ is infinite, which implies $d \rho(\tilde{X})(Z)$ is infinite by (777). So suppose $\operatorname{tr}\left(N^{s-1} V\right)=0$ for $s=2, \ldots, n$. By (53) and (60),

$$
\langle Y, Z\rangle_{\mathbb{C}^{n \times n}}=\langle W, V\rangle_{\mathbb{C}^{n \times n}}=\sum_{\ell=1}^{n} \operatorname{Re}\left(\overline{\theta_{\ell}} \operatorname{tr}\left(N^{\ell-1} V\right)\right),
$$

which simplifies to

$$
\operatorname{Re}\left(\overline{\theta_{1}} \operatorname{tr}(V)\right)=\left\langle\theta_{1}, \operatorname{tr}(V)\right\rangle_{\mathbb{C}}
$$


Therefore,

$$
\begin{aligned}
\langle Y, Z\rangle_{\mathbb{C}^{n \times n}} & =\left\langle\theta_{1}, \operatorname{tr}(V)\right\rangle_{\mathbb{C}} & & \\
& \leqslant \max _{\zeta \in \mathbb{B}}\langle\zeta / n, \operatorname{tr}(V)\rangle_{\mathbb{C}} & & \text { by (75) } \\
& =|\operatorname{tr}(V)| / n & & \\
& =\mathrm{d} \mathbf{r}\left(e_{(n, 0)}\right)(q(\lambda)) & & \text { by (78) } \\
& \leqslant \mathrm{d} \rho(\tilde{X})(Z) & & \text { by (77). }
\end{aligned}
$$

Condition (76) applied to the inequality above implies $Y \in \hat{\partial} \rho(X)$.

Now suppose $Y \in \hat{\partial} \rho(\tilde{X})$. By Theorem 15, $Y$ satisfies (50)-(53). Choose $Z:=$ $\widetilde{P}^{-1} V \widetilde{P}$ with $V:=\theta_{1} I$. By the definition of subderivative in (2.3) (using $\rho(\tilde{X})=0$ ),

$$
\begin{aligned}
\operatorname{d} \rho(\tilde{X})(Z) & =\liminf _{t \downarrow 0, \tilde{Z} \rightarrow Z} \rho(\tilde{X}+t \widetilde{Z}) / t \\
& \leqslant \liminf _{t \downarrow 0} \rho(\tilde{X}+t Z) / t \\
& =\liminf _{t \downarrow 0} \rho(N+t V) / t, \quad \text { where } N \text { is given in (56) } \\
& =\liminf _{t \downarrow 0} t\left|\theta_{1}\right| / t, \quad \text { since the only eigenvalue of } N+t V \text { is } t \theta_{1}, \\
& =\left|\theta_{1}\right| .
\end{aligned}
$$

By combining (76) and the inequality above,

$$
\left|\theta_{1}\right| \geqslant \mathrm{d} p(\tilde{X})(Z) \geqslant\langle Y, Z\rangle_{\mathbb{C}^{n \times n}}=\left\langle\widetilde{P}^{-*} Y \widetilde{P}^{*}, V\right\rangle_{\mathbb{C}^{n \times n}}=\operatorname{Re}\left(\overline{\theta_{1}} \operatorname{tr}(V)\right)=n\left|\theta_{1}\right|^{2},
$$

which implies $1 / n \geqslant\left|\theta_{1}\right|$, giving (75).

The formula for the recession cone of the regular subdifferential parallels that of Theorem 21 .

Finally, we show that nonderogatory active eigenvalues are sufficient for regularity. Suppose $Y \in \partial \rho(\tilde{X})$. Then, by Theorem 15,

$$
\widetilde{P}^{-*} Y \widetilde{P}^{*}=\sum_{\ell=1}^{n} \theta_{\ell}\left(N^{*}\right)^{\ell-1},
$$

that is, $Y$ is lower triangular Toeplitz and has only one eigenvalue, $\theta_{1}$. By definition of subgradient (13), there exists $X^{\nu} \stackrel{\nu}{\longrightarrow} \tilde{X}$ and $Y^{\nu} \in \hat{\partial} \rho\left(X^{\nu}\right)$ with $Y^{\nu} \stackrel{\nu}{\longrightarrow} Y$. Since the set of nonderogatory matrices is open in $\mathbb{C}^{n \times n}$, we may without loss in generality assume that $X^{\nu}$ is nonderogatory for all $\nu$. If there is an infinite subsequence with $\rho\left(X^{\nu}\right)=0$, then $X^{\nu}=\tilde{X}$ along this subsequence and there is nothing more to show. So suppose $\rho\left(X^{\nu}\right)>0$ for all $\nu$. Since there are only finitely many partitions of $n$, we may assume (by passing to a subsequence if necessary) that the distinct eigenvalues of $X^{\nu}$ are $\lambda_{1}^{\nu}, \ldots, \lambda_{m}^{\nu}$ with multiplicities $n_{1}, \ldots, n_{m}$ with $\lambda_{j}^{\nu} \rightarrow 0$ for $j=1, \ldots, m$ and that the set of active indices $\tilde{\mathcal{I}}:=\mathcal{I}_{\rho}\left(X^{\nu}\right)$ is constant with respect to $\nu$. By Theorem 25, there exist nonsingular $P^{\nu}$ for all $\nu$ such that the diagonal of $\left(P^{\nu}\right)^{-*} Y^{\nu}\left(P^{\nu}\right)^{*}$ equals $\left(\theta_{11}^{\nu}, \ldots, \theta_{1 n_{1}}^{\nu}, \ldots, \theta_{m 1}^{\nu}, \ldots, \theta_{m n_{m}}^{\nu}\right)$, which satisfy

$$
\theta_{j 1}^{\nu} / \lambda_{j}^{\nu} \in \mathbb{R}, \theta_{j 1} / \lambda_{j}^{\nu} \geqslant 0 \text {, for } j \in \widetilde{\mathcal{I}}, \quad \sum_{j \in \widetilde{\mathcal{I}}} n_{j} \theta_{j 1}^{\nu}\left|\lambda_{j}^{\nu}\right| / \lambda_{j}^{\nu}=1 \text {, and } \theta_{j 1}^{\nu}=0 \text { for } j \notin \widetilde{\mathcal{I}} \text {. }
$$

Recall from (74) that $\nabla r(\lambda)=\lambda /|\lambda|$. By the compactness of $\mathbb{B}$, we may assume that there exist $\phi_{j} \in \mathbb{B}$ such that $\nabla r\left(\lambda_{j}^{\nu}\right) \rightarrow \phi_{j}$ for $j \in \tilde{\mathcal{I}}$. The remainder of this proof 
follows that of [2, Theorem 7.3]. Rewrite the summation in the previous displayed equation as

using (74). Define

$$
\sum_{j \in \tilde{\mathcal{I}}} n_{j} \theta_{j 1}^{\nu} / \nabla r\left(\lambda_{j}^{\nu}\right)=1
$$

$$
\gamma_{j}^{\nu}:= \begin{cases}n_{j} \theta_{j 1}^{\nu} / \nabla r\left(\lambda_{j}^{\nu}\right), & \text { if } j \in \widetilde{\mathcal{I}}, \\ 0, & \text { if } j \notin \widetilde{\mathcal{I}} .\end{cases}
$$

Then $\left(\gamma_{1}^{\nu}, \ldots, \gamma_{m}^{\nu}\right) \in \Delta^{m-1}$, the simplex defined in (59). Since $\Delta^{m-1}$ is compact, and by passing to a subsequence if necessary, there exists $\left(\gamma_{1}, \ldots, \gamma_{m}\right) \in \Delta^{m-1}$ such that $\left(\gamma_{1}^{\nu}, \ldots, \gamma_{m}^{\nu}\right) \rightarrow\left(\gamma_{1}, \ldots, \gamma_{m}\right)$. The eigenvalues of $Y^{\nu}$ are $\theta_{11}^{\nu}, \ldots, \theta_{m 1}^{\nu}$ with multiplicities $n_{1}, \ldots, n_{m}$, and they converge to $\theta_{1}$, the only eigenvalue of $Y$. Therefore,

$$
\lim _{\nu \rightarrow \infty} \gamma_{j}^{\nu} \nabla r\left(\lambda_{j}^{\nu}\right)=\gamma_{j} \phi_{j}=n_{j} \theta_{1}
$$

for $j=1, \ldots, m$. Summing over $j$, we have

$$
\sum_{j=1}^{m} \gamma_{j} \phi_{j}=\sum_{j=1}^{m} n_{j} \theta_{1}=\theta_{1} \sum_{j=1}^{m} n_{j}=\theta_{1} n .
$$

Since $\left(\gamma_{1}, \ldots, \gamma_{m}\right) \in \Delta^{m-1}$, and $\phi_{j} \in \mathbb{B}$ for all $j$,

$$
\left|\theta_{1}\right| n=\left|\sum_{j=1}^{m} \gamma_{j} \phi_{j}\right| \leqslant \sum_{j=1}^{m} \gamma_{j}\left|\phi_{j}\right| \leqslant \sum_{j=1}^{m} \gamma_{j}=1,
$$

which implies $\left|\theta_{1}\right| \leqslant 1 / n$.

Now suppose $Y \in \partial^{\infty} \rho(\tilde{X})$. By Theorem [15, representation (79) holds. By definition of horizon subgradient (2.3), there exist $X^{\nu} \stackrel{\nu}{\longrightarrow} \tilde{X}, s^{\nu} \downarrow 0$ and $Y^{\nu} \in$ $\hat{\partial} \rho\left(X^{\nu}\right)$ with $s^{\nu} Y^{\nu} \stackrel{\nu}{\longrightarrow} Y$. Set $V:=\theta_{1} I$ and $Z:=\widetilde{P}^{-1} V \widetilde{P}$. Computing the inner product,

$$
\langle Y,(\tilde{X}+t Z)-\tilde{X}\rangle_{\mathbb{C}^{n \times n}}=t\langle Y, Z\rangle_{\mathbb{C}^{n \times n}}=t\langle W, V\rangle_{\mathbb{C}^{n \times n}}=t n\left|\theta_{1}\right|^{2} .
$$

Since $Y^{\nu} \in \hat{\partial} \rho\left(X^{\nu}\right)$,

$$
\rho\left(X^{\nu}+t Z\right)-\rho\left(X^{\nu}\right) \geqslant\left\langle Y^{\nu},\left(X^{\nu}+t Z\right)-X^{\nu}\right\rangle_{\mathbb{C}^{n \times n}}+o(\|t Z\|),
$$

for all $t>0$, which implies

$$
s^{\nu}\left(\rho\left(X^{\nu}+t Z\right)-\rho\left(X^{\nu}\right)\right) \geqslant\left\langle s^{\nu} Y^{\nu},\left(X^{\nu}+t Z\right)-X^{\nu}\right\rangle_{\mathbb{C}^{n \times n}}+s^{\nu} o(\|t Z\|)
$$

for all $t>0$. Taking the limit as $\nu \rightarrow \infty$ gives

$$
\begin{aligned}
& 0 \geqslant\langle Y,(\tilde{X}+t Z)-\tilde{X}\rangle_{\mathbb{C}^{n \times n}}, \quad \text { which, by (80), implies } \\
& 0 \geqslant t n\left|\theta_{1}\right|^{2} \geqslant 0,
\end{aligned}
$$

that is, $\theta_{1}=0$.

Theorem 28 (Derogatory active eigenvalues). Suppose $\tilde{X} \in \mathbb{C}^{n \times n}$ has a derogatory active eigenvalue. Then $\partial \rho(\tilde{X}) \supsetneq \hat{\partial} \rho(\tilde{X})$.

Proof. Let $\tilde{X} \in \mathbb{C}^{n \times n}$. For the case $\rho(\tilde{X})>0$, see the proof of Theorem 22 . Suppose $\rho(\tilde{X})=0$ and that the eigenvalue 0 is derogatory. Let $\varepsilon^{\nu} \downarrow 0$ and set $X^{\nu}:=$ $\widetilde{P}\left(J+\varepsilon^{\nu} E\right) \widetilde{P}^{-1}$, where $J$ and $\widetilde{P}$ are defined in (34) (with the index $j$ suppressed and where the matrix $\widetilde{B}$ does not appear) and $E$ is one in the first $m_{1}$ diagonal positions corresponding to block $J_{1}$ and is zero elsewhere. The matrix $X^{\nu}$ has exactly one active eigenvalue, $\varepsilon^{\nu}$, with multiplicity $m_{1}$, and $\widetilde{P}\left(J+\varepsilon^{\nu} E\right) \widetilde{P}^{-1}$ is the Jordan form of $X^{\nu}$. By Theorem 25, $\hat{\partial} \rho\left(X^{\nu}\right)$ includes the matrix $\left(1 / m_{1}\right) \widetilde{P}^{-*} E \widetilde{P}^{*}$ for all $\nu$. 
Therefore, $\left(1 / m_{1}\right) \widetilde{P}^{-*} E \widetilde{P}^{*} \in \partial \rho(\tilde{X})$. Yet by Theorem 15 , $\left(1 / m_{1}\right) \widetilde{P}^{-*} E \widetilde{P}^{*} \notin \hat{\partial} \rho(\tilde{X})$ since its diagonal entries are zero for blocks $m_{2}$ to $m_{q}$ but nonzero for block $m_{1}$, i.e. the diagonal entries are not equal. Therefore, $\partial \rho(\tilde{X}) \supsetneq \hat{\partial} \rho(\tilde{X})$ if $\tilde{X}$ has a derogatory active eigenvalue.

Theorem 29. The spectral radius is subdifferentially regular at $\tilde{X} \in \mathbb{C}^{n \times n}$ if and only if all active eigenvalues of $\tilde{X}$ are nonderogatory.

7.1. Examples. First consider the matrix

$$
A=\left[\begin{array}{ccc}
1 & 1 & 0 \\
0 & 1 & 0 \\
0 & 0 & -1
\end{array}\right],
$$

which has two nonderogatory active eigenvalues, 1 and -1 . By Theorem 25, $Y \in$ $\partial \rho(A)$ if and only if there exist $\theta_{11}, \theta_{12}, \theta_{21} \in \mathbb{C}$ such that

$$
Y=\left[\begin{array}{ccc}
\theta_{11} & 0 & 0 \\
\theta_{12} & \theta_{11} & 0 \\
0 & 0 & \theta_{21}
\end{array}\right],
$$

where $\operatorname{Re}\left(\theta_{12}\right) \geqslant-\theta_{11}, \quad \theta_{11} \in[0, \infty), \quad \theta_{21} \in(-\infty, 0], \quad$ and $2 \theta_{11}-\theta_{21}=1$. Second consider the matrix

$$
B=\left[\begin{array}{lll}
1 & 1 & 0 \\
0 & 1 & 0 \\
0 & 0 & 1
\end{array}\right] .
$$

By Theorem 25, $Y \in \hat{\partial} \rho(B)$ if and only if

$$
Y=\frac{1}{3} I+\left[\begin{array}{lll}
0 & 0 & 0 \\
\theta & 0 & 0 \\
0 & 0 & 0
\end{array}\right],
$$

where $\operatorname{Re}(\theta) \geqslant-1 / 3$. Next consider the sequence of matrices

$$
B^{\nu}:=B+\operatorname{Diag}(0,0,1 / \nu)
$$

for $\nu \in \mathbb{N}$. The only active eigenvalue of $B^{\nu}$ is $(1+1 / \nu)$, which is nonderogatory. By Theorem 25] $M:=\operatorname{Diag}(0,0,1) \in \hat{\partial} \rho\left(B^{\nu}\right)$ for all $\nu$, which implies $M \in \partial \rho(B)$, yet $M \notin \hat{\partial} \rho(B)$ since it does not satisfy the form given in (81), i.e. the diagonal entries are not equal.

\section{Summary}

This paper extends the variational results for the spectral abscissa mapping in 11] to convexly generated spectral max functions. Two very different methods of analysis are applied. The first uses the Arnold form (4.1) and tools from [5]. A nonsmooth chain rule is applied to the composition of the characteristic polynomial mapping and a max root function for polynomials (6). The subdifferential theory for polynomial max root functions is developed in 2, which in turn builds on the work in [8, 9. The key technical breakthroughs for the first approach appear in 44.2 culminating in Theorem 10, which describes the variational behavior of the mapping $G$ taking a matrix to its distinct active monomial factors on the space $\mathbb{S}_{\tilde{p}}$. This yields our first main result, Theorem 12, which gives a formula for the

\footnotetext{
${ }^{2}$ The matrix $B-I$ is in the family of matrices considered in 14 for the spectral abscissa.
} 
subdifferential and establishes subdifferential regularity when all active eigenvalues are nonderogatory.

The major drawback to Theorem 12 is the requirement that all of the active eigenvalues be nonderogatory. It is this hypothesis that gives access to our polynomial results through Theorem 10, Our second line of attack avoids the polynomial results by appealing directly to underlying matrix structures. This approach extends results in 11 to convexly generated spectral max functions when possible. In Theorem [19, we characterize the regular subgradients of a convexly generated spectral max function without assuming nonderogatory active eigenvalues. In addition, Lemma 22 shows that nonderogatory active eigenvalues are necessary for subdifferential regularity. Combined with our earlier results, we obtain Theorem 23. which shows that subdifferential regularity occurs if and only if all active eigenvalues are nonderogatory. This neatly extends the 2001 result of Burke and Overton for the spectral abscissa [1] to the class of convexly generated spectral max functions satisfying (3).

The results are applied in Section 7 to obtain new variational properties for the spectral radius mapping.

\section{REFERENCES}

[1] V.I. Arnold. On matrices depending on parameters. Russian Mathematical Surveys, 26(2):2943, 1971.

[2] J.V. Burke and J. Eaton. On the subdifferential regularity of max root functions for polynomials. Nonlinear Analysis: Theory, Methods and Applications, 75(3):1168-1187, 2012.

[3] J.V. Burke, D. Henrion, A.S. Lewis, and M.L. Overton. Stabilization via nonsmooth, nonconvex optimization. IEEE Transactions on Automatic Control, 51(11):1760-1769, 2006.

[4] J.V. Burke, A.S. Lewis, and M.L. Overton. Optimizing matrix stability. Proceedings of the American Mathematical Society, 129(6):1635-1642, 2000.

[5] J.V. Burke, A.S. Lewis, and M.L. Overton. Optimal stability and eigenvalue multiplicity. Foundations of Computational Mathematics, 1(2):205-225, 2001.

[6] J.V. Burke, A.S. Lewis, and M.L. Overton. Two numerical methods for optimizing matrix stability. Linear Algebra and its Applications, 351-352:117-145, 2002.

[7] J.V. Burke, A.S. Lewis, and M.L. Overton. Robust stability and a criss-cross algorithm for pseudospectra. IMA Journal of Numerical Analysis, 23:359-375, 2003.

[8] J.V. Burke, A.S. Lewis, and M.L. Overton. Variational analysis of the abscissa mapping for polynomials via the Gauss-Lucas Theorem. Journal of Global Optimization, 28(3):259-268, 2004.

[9] J.V. Burke, A.S. Lewis, and M.L. Overton. Variational analysis of functions of the roots of polynomials. Mathematical Programming Series B, 104(2):263-292, 2005.

[10] J.V. Burke and M.L. Overton. Differential properties of the spectral abscissa and the spectral radius for analytic matrix-valued mappings. Nonlinear Analysis: Theory, Methods 8 Applications, 23(4):467-488, 1994.

[11] J.V. Burke and M.L. Overton. Variational analysis of non-Lipschitz spectral functions. Mathematical Programming Series A, 90(2):317-351, 2001.

[12] J.V. Burke and M.L. Overton. Variational analysis of the abscissa mapping for polynomials. SIAM Journal on Control and Optimization, 39(6):1651-1676, 2001.

[13] F.H. Clarke. Optimization and Nonsmooth Analysis. Republished by SIAM, Philadelphia, 1990.

[14] S. Grundel and M.L. Overton. Variational analysis of the spectral abscissa at a matrix with a nongeneric multiple eigenvalue. Set-Valued and Variational Analysis, 22(1):19-43, 2014.

[15] P.D. Lax. Linear Algebra. John Wiley, New York, 1997.

[16] A.S. Lewis. Nonsmooth analysis of eigenvalues. Mathematical Programming Series A, 54(1):1-24, 1999.

[17] A.S. Lewis. The mathematics of eigenvalue optimization. Mathematical Programming Series B, 97(1):155-176, 2003. 
VARIATIONAL ANALYSIS OF CONVEXLY GENERATED SPECTRAL MAX FUNCTIONS 27

[18] B.S. Mordukhovich. Variational Analysis and Generalized Differentiation: Vol. 1. Springer, New York, 2005.

[19] R.T. Rockafellar and R.J.B. Wets. Variational Analysis. Springer, Berlin, 1998.

University of Washington, Department of Mathematics, Box 354350 Seattle, WashINGTON 98195-4350 (JVBURKE@UW.EDU)

University of Washington Tacoma, School of Interdisciplinary Arts and Sciences, Box 358436, TACOMA, WA 98402 (JREATON@Uw.EDU) 